\title{
Pharmacologic inhibition of fatty acid oxidation sensitizes human leukemia cells to apoptosis induction
}

\author{
Ismael Samudio, ${ }^{1}$ Romain Harmancey, ${ }^{2}$ Michael Fiegl, ${ }^{1}$ Hagop Kantarjian, ${ }^{3}$ \\ Marina Konopleva, ${ }^{3}$ Borys Korchin, ${ }^{1}$ Kumar Kaluarachchi, ${ }^{4}$ William Bornmann, ${ }^{4}$ \\ Seshagiri Duvvuri, ${ }^{1}$ Heinrich Taegtmeyer, ${ }^{2}$ and Michael Andreeff 1,3
}

\begin{abstract}
'Section of Molecular Hematology and Therapy, Department of Stem Cell Transplantation and Cellular Therapy, University of Texas M.D. Anderson Cancer Center, Houston, Texas, USA. ${ }^{2}$ Division of Cardiology, University of Texas Houston Medical School, Houston, Texas, USA. ${ }^{3}$ Department of Leukemia and ${ }^{4}$ Department of Experimental Therapeutics, University of Texas M.D. Anderson Cancer Center, Houston, Texas, USA.
\end{abstract}

\begin{abstract}
The traditional view is that cancer cells predominately produce ATP by glycolysis, rather than by oxidation of energy-providing substrates. Mitochondrial uncoupling - the continuing reduction of oxygen without ATP synthesis - has recently been shown in leukemia cells to circumvent the ability of oxygen to inhibit glycolysis, and may promote the metabolic preference for glycolysis by shifting from pyruvate oxidation to fatty acid oxidation (FAO). Here we have demonstrated that pharmacologic inhibition of FAO with etomoxir or ranolazine inhibited proliferation and sensitized human leukemia cells - cultured alone or on bone marrow stromal cells - to apoptosis induction by ABT-737, a molecule that releases proapoptotic Bcl-2 proteins such as Bak from antiapoptotic family members. Likewise, treatment with the fatty acid synthase/lipolysis inhibitor orlistat also sensitized leukemia cells to ABT-737, which supports the notion that fatty acids promote cell survival. Mechanistically, we generated evidence suggesting that FAO regulates the activity of Bak-dependent mitochondrial permeability transition. Importantly, etomoxir decreased the number of quiescent leukemia progenitor cells in approximately $50 \%$ of primary human acute myeloid leukemia samples and, when combined with either ABT-737 or cytosine arabinoside, provided substantial therapeutic benefit in a murine model of leukemia. The results support the concept of FAO inhibitors as a therapeutic strategy in hematological malignancies.
\end{abstract}

\section{Introduction}

More than half a century ago, Otto Warburg proposed that the origin of cancer cells was closely linked to a permanent respiratory defect that circumvents the Pasteur effect, i.e., the inhibition of anaerobic fermentation by oxygen (1). However, we have recently demonstrated that in leukemia cells, mitochondrial uncoupling - the continuing reduction of oxygen without the synthesis of ATP - could mimic the Warburg effect in the absence of permanent, transmissible alterations to the oxidative capacity of cells (2). This metabolic pattern was observed when leukemia cells were cultured on feeder layers of bone marrow-derived mesenchymal stromal cells (MSCs). MSCs have previously been reported to support both normal and malignant hematopoiesis (reviewed in refs. 3-5) and have become an important component in the in vitro modeling of the bone marrow microenvironment. Leukemia cells cultured on MSC feeder layers demonstrated increased lactate generation, and, most curiously, decreased mitochondrial membrane potential in the presence of a transient (6-8 hour) increase in oxygen consumption. Additionally, this uncoupled phenotype appeared to be associated with the antiapoptotic effect of MSC feeder layers, and we hypothesized a shift away from the complete oxidation of glucose. This concept has already been alluded to by Lynen (6), and by Ronzoni and Ehrenfest in experiments using the prototypical protonophore 2,4-dinitrophenol, and suggests a metabolic shift to fatty acid oxidation (FAO) rather than pyruvate oxidation $(2,7)$. Although increased FAO has been shown to

Conflict of interest: The authors have declared that no conflict of interest exists. Citation for this article: J. Clin. Invest. doi:10.1172/JCI38942. promote chemoresistance (8), to our knowledge, the therapeutic value of modulating this metabolic pathway in leukemia has not previously been investigated.

In light of this, one also must consider pyruvate (derived from glycolysis) and/or $\alpha$-ketoglutarate (derived from glutaminolysis) as anaplerotic substrates for efficient Krebs cycle use of fatty acidderived acetyl CoA (9), suggesting the possibility that in certain cell types, high rates of aerobic glycolysis and/or glutaminolysis may promote efficient FAO (i.e., fats burn in the fire of carbohydrates; ref. 10). Additionally, it has been reported that in glioma cells, approximately $60 \%$ of carbon skeletons from glucose are used for de novo fatty acid synthesis (FAS), which suggests that glycolysis may also be supporting FAO by contributing to the fatty acid pool. Figure 1A illustrates some of the relevant metabolic pathways that interact with the Krebs cycle, including the suggested role of uncoupling protein-2 (UCP2) in facilitating glutamine oxidation (11). The above observations suggest that, far from indicating a defect in mitochondrial respiration, the Warburg effect may in fact include a scenario in which high rates of aerobic glycolysis are necessary to support the mitochondrial metabolism of fatty acids.

Pharmacologic inhibition of FAO with etomoxir (EX), which inhibits the entry of fatty acids into the mitochondria by blocking the activity of carnitine palmitoyl transferase 1 (CPT1), has yielded therapeutic benefits for the treatment of heart failure by shifting the failing heart's energy supply from fatty acids to the energetically more efficient pyruvate (reviewed in ref. 12). It is thus intriguing to contemplate the possibility that, like dichloroacetate, which activates pyruvate dehydrogenase (13), EX would be cytotoxic to cancer cells by promoting the mitochondrial oxidation of pyruvate. 


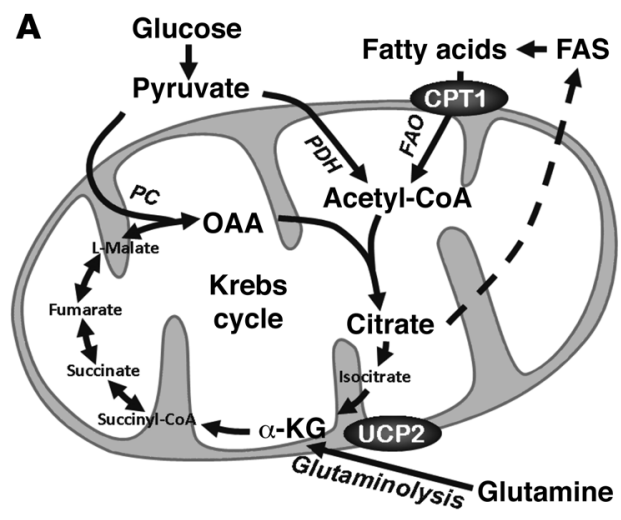

D

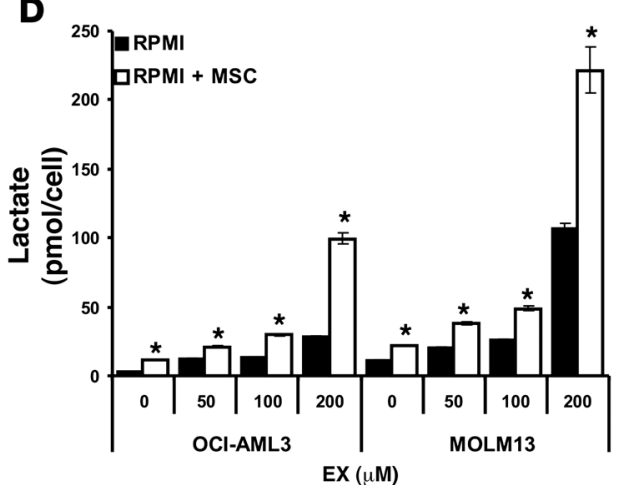

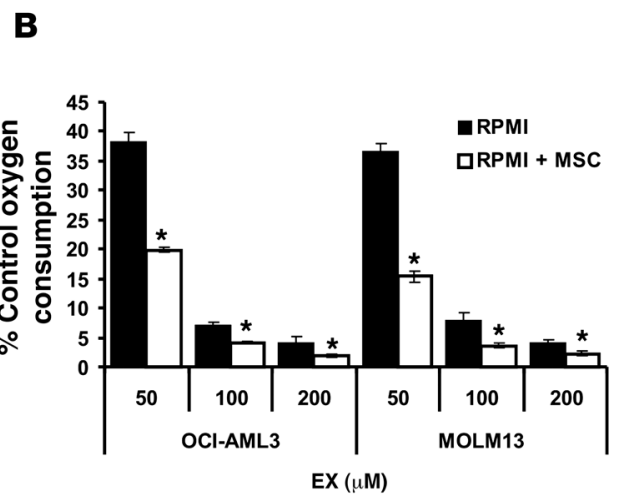
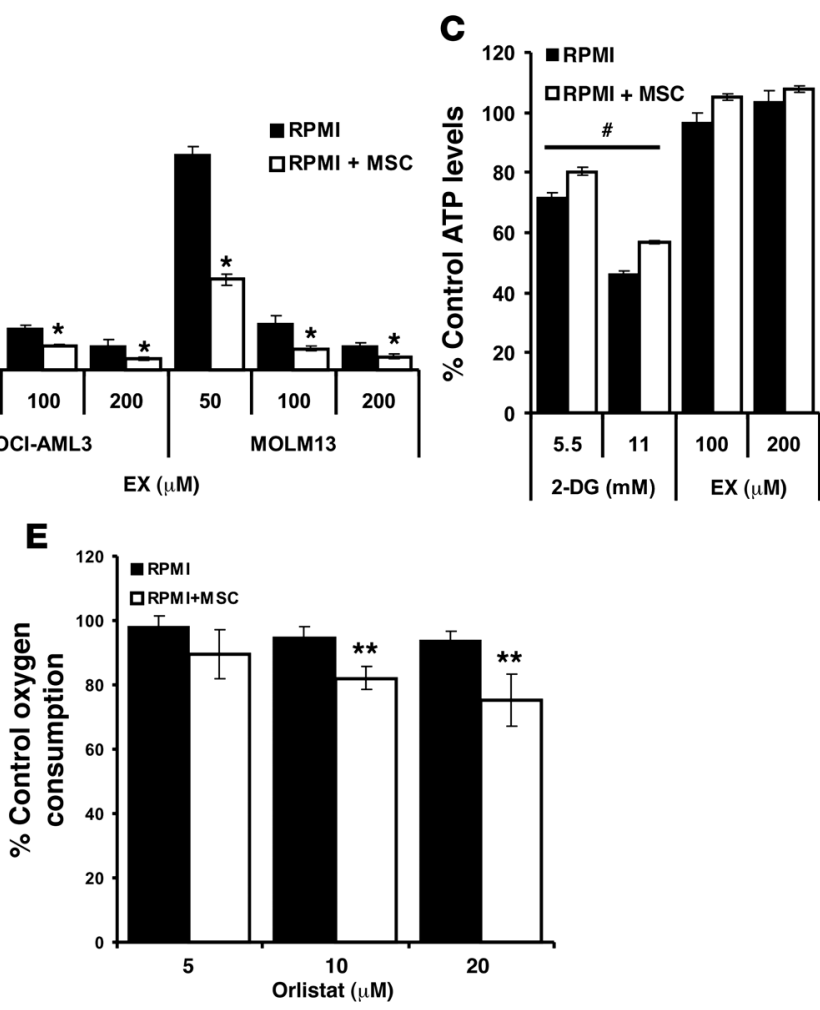

Figure 1

Leukemia cells uncouple FAO from ATP synthesis and rely on de novo FAS to support FAO. (A) Schematic representation of the relevant metabolic pathways investigated. $\alpha-K G, \alpha$-ketoglutarate; PC, pyruvate carboxylase; PDH, pyruvate dehydrogenase. (B) OCI-AML3 and MOLM13 cells were grown alone or on MSC feeder layers as described in Methods, and after MACS depletion of MSCs, $2 \times 10^{5}$ leukemia cells/well were treated with increasing concentrations of EX and monitored for oxygen consumption in Oxygen Biosensor plates for 3 hours. (C) OCI-AML3 cells were grown as in B and treated with 2-DG (5.5 and $11 \mathrm{mmol} / \mathrm{l})$ or EX (100 and $200 \mu \mathrm{mol} / \mathrm{l})$ for 6 hours, and after MACS depletion of MSCs, $2 \times 10^{5}$ leukemia cells were lysed, and ATP levels were quantitated as described in Methods. (D) OCI-AML3 and MOLM13 cells were grown alone or on MSC feeder layers and exposed to increasing concentrations of EX for 48 hours. The number of viable cells (Annexin V-negative; tetramethylrhodamine methyl ester-positive) and lactate levels were quantitated as described in Methods; results are expressed as picomoles lactate per viable cell. (E) Leukemia cells were cultured as in $\mathbf{B}$, treated with increasing concentrations of orlistat, and monitored for oxygen consumption in Oxygen Biosensor plates for 3 hours. ${ }^{\star} P<0.005,{ }^{\star \star} P<0.01$ versus monocultures; ${ }^{\#} P<0.005$ versus control.

Conversely, pharmacologic inhibition of FAO results in increased nonoxidative fatty acid metabolism, including the generation of ceramide (14), and potentiation of 2-deoxyglucose cytotoxicity (15), which suggests that FAO inhibition may decrease cell survival in the absence of increased pyruvate oxidation or decreased Krebs cycle activity. Furthermore, the truncated form of the proapoptotic Bcl-2 family member Bid (t-Bid) directly inhibits CPT1 activity, an effect antagonized by Bcl-2 overexpression (16), and CPT1 has been reported to associate with $\mathrm{Bcl}-2$ (17), suggesting that the entry of fatty acids into the mitochondria may be directly linked to the Bcl-2 apoptotic rheostat. Notably, we have recently described that antagonism of Bcl-2 using ABT-737, a BH3 mimetic that disrupts the sequestration of $\mathrm{Bax}, \mathrm{Bak}$, and other proapoptotic $\mathrm{Bcl}-2$ proteins by antiapoptotic Bcl-2 family members, induces apoptosis in leukemia cell lines and primary samples (18). However, to our knowledge, the effect of FAO inhibition on apoptosis induction by Bcl-2 antagonists in leukemia cells has thus far not been investigated.

Here we report that leukemia cells, alone or in coculture with MSCs, displayed uncoupling of fatty acid-dependent oxygen consumption from ATP synthesis and that pharmacological inhibition of FAO decreased proliferation and sensitized leukemia cells to apoptosis induced by ABT-737 and Nutlin 3a. Our results suggest that leukemia cells demonstrate a strong dependence on glycolysis for ATP generation, whereas uncoupled FAO - augmented by MSC coculture, and supported by de novo FAS and lipolysis - opposes the formation of Bak-dependent mitochondrial permeability transition. We also present evidence that the combination of EX with ABT-737 or cytosine arabinoside (Ara-C) provided therapeutic benefit in a murine leukemia model. In addition, we showed that EX decreased the number of quiescent leukemia progenitor cells (QLPs) in peripheral blood or bone marrow samples from acute myeloid leukemia (AML) patients. Our results lend support to the clinical evaluation of FAO inhibitors for the treatment of leukemia and suggest that fatty acid metabolism is intimately linked to leukemia cell apoptosis and proliferation.

\section{Results}

Leukemia cells uncouple the oxidation of fatty acids from ATP synthesis. We have previously shown that mitochondrial uncoupling can promote the Warburg effect in leukemia cells, and hypothesized that this may indicate a shift to FAO (2). To further test this hypothesis, we first investigated how pharmacological inhibition of FAO with 
A
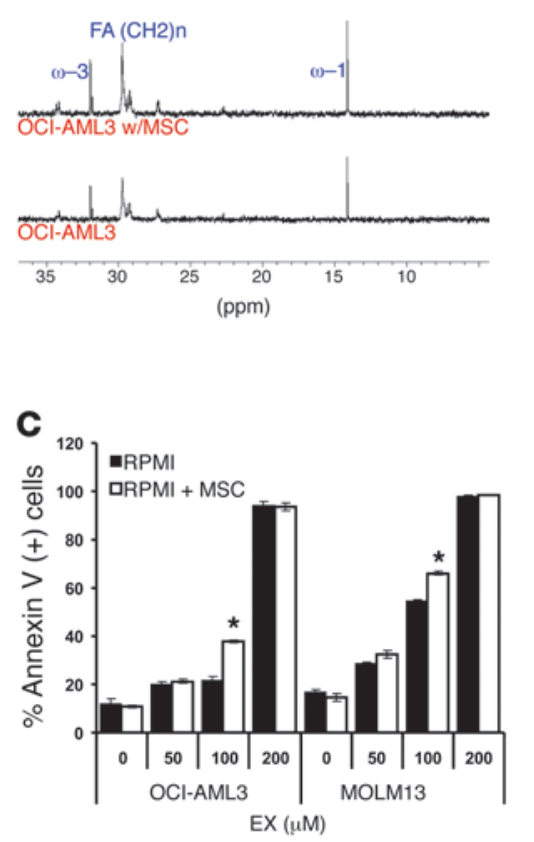

B

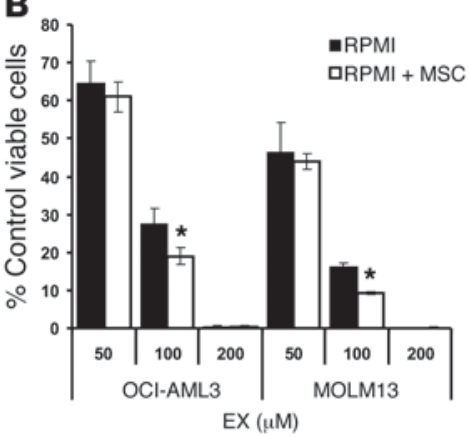

D

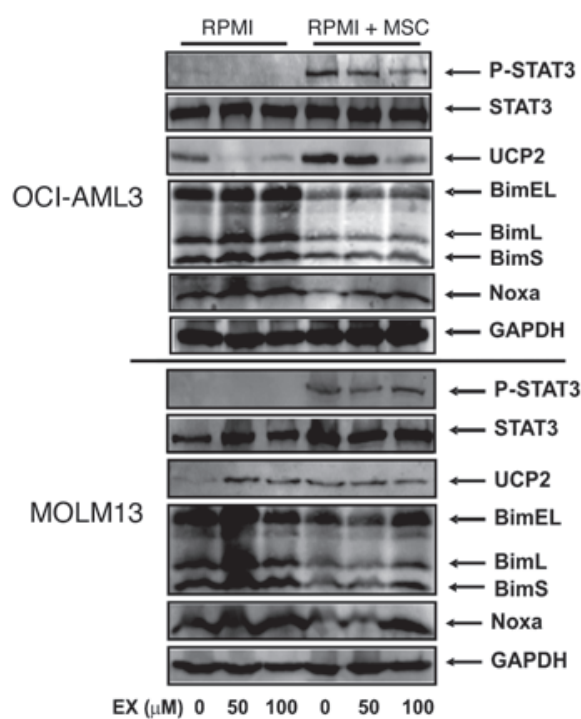

Figure 2

Pharmacological inhibition of FAO decreases proliferation of leukemia cells cultured on MSC feeder layers. (A) OClAML3 cells were grown alone or on MSC feeder layers in the presence of $11 \mathrm{mmol} / \mathrm{l}$ $\left[1-{ }^{13} \mathrm{C}\right]$ glucose for 48 hours. Lipids were extracted, and ${ }^{13} \mathrm{C}$ spectra were acquired as described in Methods. (B) OCl-AML3 and MOLM13 cells were grown alone or on MSC feeder layers and exposed to increasing concentrations of EX for 96 hours. The number of viable cells was quantitated by flow cytometry as described in Methods. (C) Cells were cultured as in B, and the percent Annexin V-positive cells was quantitated by flow cytometry as described in Methods. (D) Monocultures and MSC cocultures of OCI-AML3 and MOLM13 cells were treated with EX for 24 hours, and after MACS depletion of MSCs, cell extracts were immunoblotted as described in Methods. ${ }^{*} P<0.05$ versus monocultures.
EX affected oxygen consumption in OCI-AML3 and MOLM13 cells alone or cultured in MSC feeder layers. As shown in Figure 1B, treatment with EX for 3 hours inhibited oxygen consumption in OCI-AML3 and MOLM13 cells cultured alone, and this inhibitory effect was significantly more pronounced $(P<0.01)$ for all doses of this agent in coculture. These observations suggest that MSC feeder layers increased the dependence of oxygen consumption on FAO in leukemia cells. To investigate the contribution of FAO to energy metabolism in leukemia cells, we treated OCI-AML3 cells cultured alone or on stroma feeder layers with the glycolysis inhibitor 2-deoxyglucose (2-DG) or EX for 6 hours. We found that 2-DG, but not EX, decreased ATP levels in OCI-AML3 cells alone and in coculture (Figure 1C). Similar results were observed in MOLM13 cells cultured on MSC feeder layers (data not shown) and in U937 cells in monoculture (Supplemental Figure 1; supplemental material available online with this article; doi:10.1172/JCI38942DS1). Likewise, the piperazine derivative ranolazine - which partially inhibits the terminal enzyme in FAO, 3-ketoacyl CoA thiolase (19) - also abrogated oxygen consumption, but did not decrease ATP levels in OCI-AML3 cells and a primary leukemia sample cultured alone (Supplemental Figure 2). This result suggests that the observed effects on oxygen consumption are mediated by FAO per se, and not by the entry of fatty acids into the mitochondrial matrix. Taken together, these findings indicate that leukemia cells actively reduce oxygen using electrons derived from FAO, that FAO does not contribute to ATP synthesis, and that MSC coculture augments this metabolic pattern.
Lenkemia cells rely on de novo FAS. Because EX has been reported to increase the oxidation of pyruvate in cardiomyocytes (20), we next investigated whether EX decreases generation of lactate in OCI-AML3 and MOLM13 cells cultured alone or on MSC feeder layers. Interestingly, after 48 hours of treatment, EX promoted a dose-dependent increase in the accumulation of lactate in the culture medium in cells alone or in coculture (Figure 1D), which suggests that in leukemia cells, FAO inhibition does not promote pyruvate oxidation. Although inhibition of FAO does not affect ATP pools, the observed increase in glycolytic activity is likely to be an adaptive mechanism to counteract any decrease in ATP production (Supplemental Figure 3). A similar effect was observed with ranolazine in OCI-AML3 cells and a primary leukemia sample cultured alone (Supplemental Figure 4). Of note, OCI-AML3 cells showed minimal metabolism of exogenous oleate compared with MSCs alone and appeared to inhibit oleate metabolism by MSCs (Supplemental Figure 5). Because leukemia cells have been shown to express fatty acid synthase (21), we investigated the contribution of FAS to oxygen consumption. For this experiment, we cultured OCI-AML3 cells alone or on MSC feeder layers and exposed them to increasing concentrations of the fatty acid synthase/lipase inhibitor orlistat (22). As shown in Figure 1E, 3 hours of treatment with orlistat decreased oxygen consumption in a dose-dependent manner in OCI-AML3 cells cultured on MSCs, but did not significantly inhibit oxygen consumption in monocultures; however, longer incubations (more than 12 hours) decreased oxygen consumption in OCI-AML3 cells cultured alone (data not shown). 


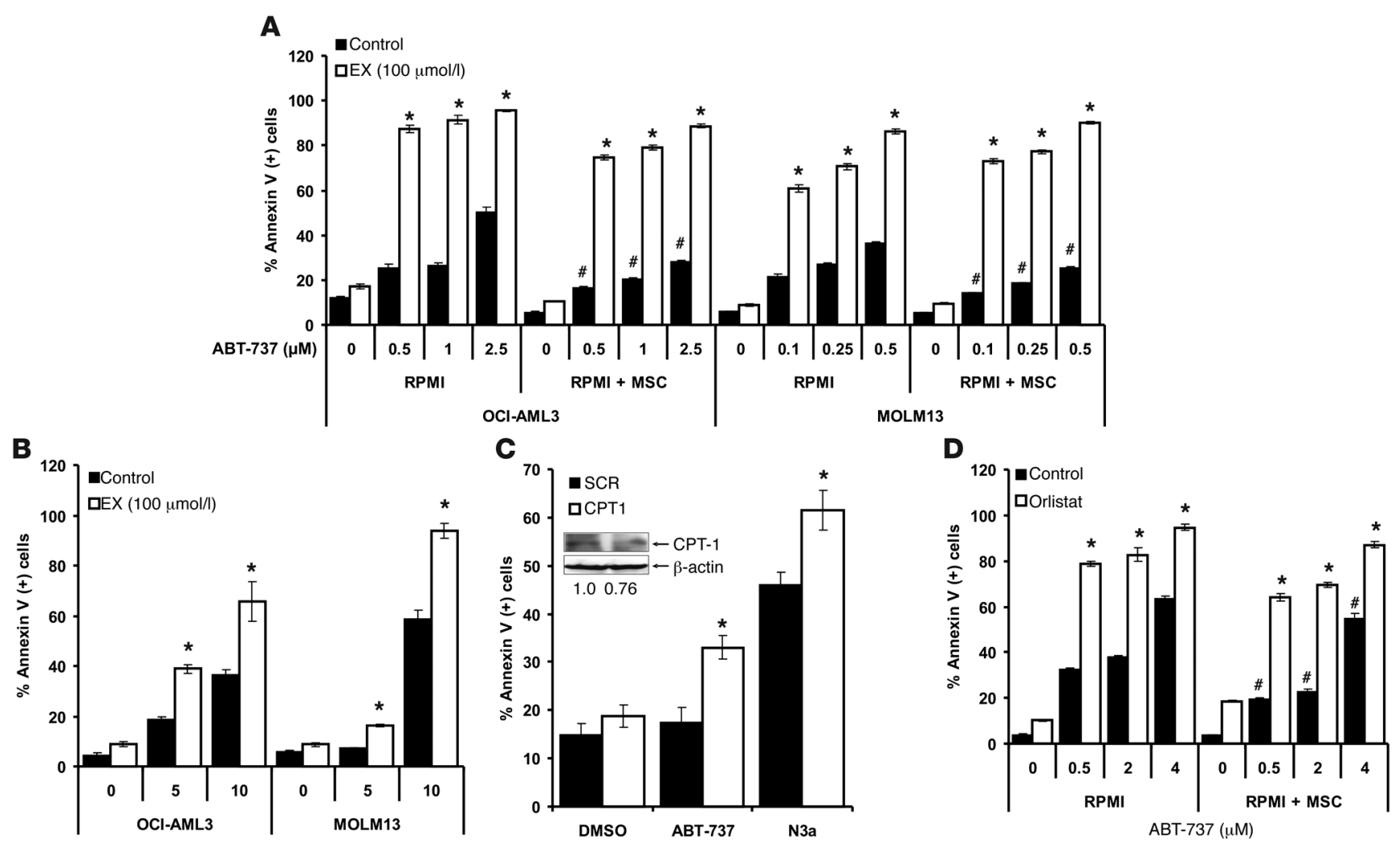

Figure 3

Pharmacologic or genetic manipulation of $\beta$-oxidation sensitizes leukemia cells to apoptosis induced by ABT-737 or Nutlin 3a. (A) Monocultures and MSC cocultures of OCl-AML3 and MOLM13 cells were exposed to $100 \mu \mathrm{mol} / \mathrm{I} \mathrm{EX}$ alone or in combination with increasing concentrations of ABT-737 for 24 hours, and the percent Annexin V-positive cells was quantitated by flow cytometry as described in Methods. ${ }^{\star} P<0.0001$ versus control; $\# P<0.01$ versus monocultures. (B) Monocultures of leukemia cells were exposed to $100 \mu \mathrm{mol} / \mathrm{I} E X$ alone or in combination with increasing doses of Nutlin 3a for 24 (MOLM13) or 48 (OCl-AML3) hours, and the percent Annexin V-positive cells was quantitated by flow cytometry. ${ }^{\star} P<0.001$ versus control. (C) OCl-AML3 cells were electroporated with siRNA duplexes targeting CPT1 or scrambled control (SCR) duplexes as described in Methods. At 16 hours after nucleofection, cells were treated with $2 \mu \mathrm{mol} / \mathrm{I}$ ABT-737 or $10 \mu \mathrm{mol} / \mathrm{l}$ Nutlin $3 a$ (N3a) for 24 hours, and apoptosis was analyzed by flow cytometry as described in Methods. ${ }^{*} P<0.01$ versus scrambled siRNA. In parallel, the expression of CPT1 and $\beta$-actin in untreated SCR and CPT1 siRNA nucleofected cells was quantitated by immunoblotting as described in Methods. (D) OCI-AML3 cells alone or in coculture with MSCs were treated with $10 \mu \mathrm{M}$ orlistat alone or in combination with increasing doses of ABT-737 for 24 hours, and the percent Annexin V-positive cells was quantitated by flow cytometry. ${ }^{*} P<0.0001$ versus control; ${ }^{*} P<0.01$ versus monocultures.

The above observations are biologically significant because they suggest that FAS and/or lipolysis support FAO in leukemia cells. Furthermore, ${ }^{13} \mathrm{C}-\mathrm{NMR}$ analysis suggested that OCI-AML3 cells cultured alone - and, to a greater extent, OCI-AML3 cells grown on MSC feeder layers (about 30\%-40\% more) - incorporated ${ }^{13} \mathrm{C}$ from $\left[1-{ }^{13} \mathrm{C}\right]$ glucose into $\omega-1, \omega-3$, and total fatty acids (Figure $2 \mathrm{~A}$ ). Taken together, the results illustrate that leukemia cells grown on MSC feeder layers rely on high rates of glycolysis to supply carbon skeletons for de novo FAS, and that de novo FAS and/or lipolysis in turn provides substrates to support FAO.

Pharmacological inhibition of FAO decreases proliferation of leukemia cells cultured on MSC feeder layers. Because the contribution of FAO to the proliferation of leukemia cells on MSC feeder layers had not to our knowledge been investigated before, we exposed OCI-AML3 and MOLM13 cells to increasing concentrations of $\operatorname{EX}(0,50,100$, and $200 \mu \mathrm{M}$ ) for 96 hours - alone or cultured on MSC feeder layers - and quantitated the number of viable cells. As shown in Figure 2B, EX markedly decreased the number of viable cells in a dose-dependent manner in both OCI-AML3 and MOLM13 cells grown alone and on MSC feeder layers, with $\mathrm{IC}_{50}$ values of 64.1,
60.4, 54.6, and $51.4 \mu \mathrm{mol} / 1$ for OCI-AML3, OCI-AML3 on MSCs, MOLM13, and MOLM13 on MSCs, respectively. Notably, EX and ranolazine also inhibited growth of monocultures of U937 cells (p53 mutated; Supplemental Figure 6), which suggests that the antiproliferative effects of FAO inhibitors is independent of p53; similar results were seen in HL60 cells (p53 null; data not shown). To investigate the contribution of apoptosis to the observed antileukemic effect, we used flow cytometry to quantitate the externalization of phosphatidyl serine in OCI-AML3 and MOLM13 cells alone or cultured on MSC feeder layers and treated with EX for 96 hours. EX induced apoptosis in a dose-dependent manner (Figure 2C), although the $\mathrm{EC}_{50}$ values were significantly $(P<0.001)$ higher than those required for the observed antiproliferative effects $(145,123,115$, and $97 \mu \mathrm{mol} / 1$ for OCI-AML3, OCI-AML3 on MSCs, MOLM13, and MOLM13 on MSCs, respectively), which suggests that other growth-inhibitory mechanisms may be at play. Interestingly, the $\mathrm{EC}_{50}$ values for apoptosis induction by $\mathrm{EX}$ indicate a moderate, albeit not statistically significant, decrease in OCI-AML3 and MOLM13 grown on MSC feeder layers, suggesting the possibility that fatty acid metabolism in MSC cocultures 
A

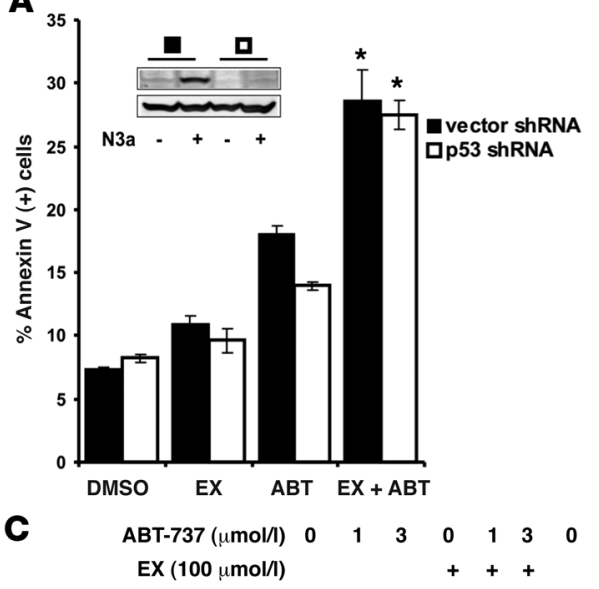

B
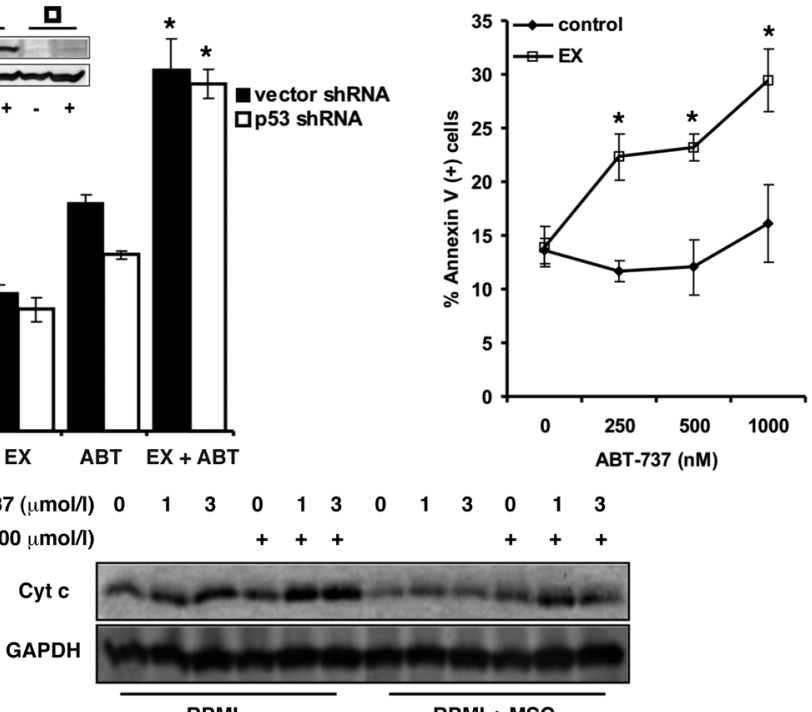

RPMI

RPMI + MSC

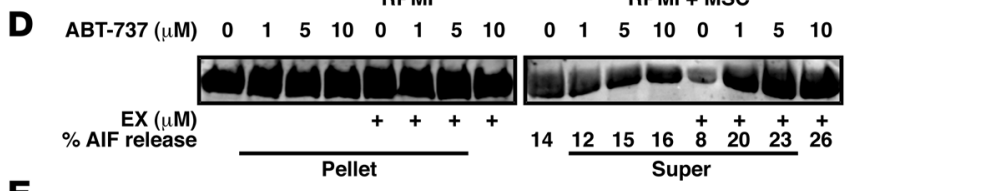

E

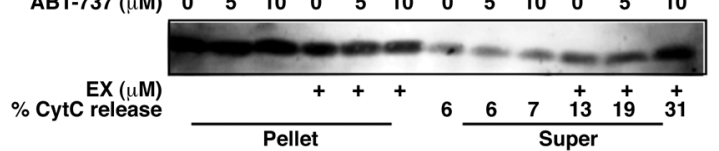

$\mathbf{F}$

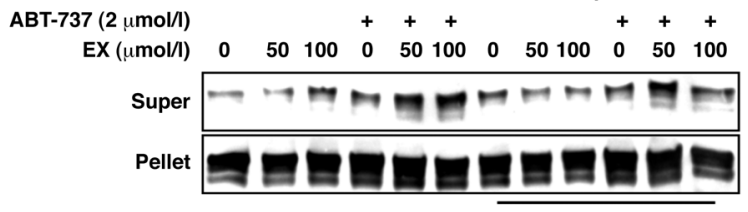

w/MSC

$\begin{array}{lllllllllllll}\% \text { AlF release } & 3 & 11 & 26 & 25 & 36 & 39 & 25 & 19 & 16 & 26 & 39 & 31\end{array}$

\section{Figure 4}

Inhibition of FAO facilitates mitochondrial permeability transition after ABT-737 treatment independently of p53. (A) OCI-AML3 cells expressing a shRNA targeting p53 or their vector control counterparts were treated with $100 \mu \mathrm{mol} / \mathrm{I} \mathrm{EX}$ alone or in combination with $2 \mu \mathrm{mol} / \mathrm{I}$ ABT-737 for 24 hours. Apoptosis was quantitated as described in Methods. Inset shows a representative Western blot of p53 and $\beta$-actin from lysates of p53 shRNA and vector shRNA cells treated with $5 \mu \mathrm{mol} / \mathrm{l}$ Nutlin $3 a$ for 6 hours. (B) U937 cells were treated with $100 \mu \mathrm{mol} / \mathrm{I}$ EX alone or in combination with increasing doses of ABT737 for 24 hours. Apoptosis was analyzed as above. (A and $\mathbf{B}){ }^{*} P<0.005$ versus ABT-737 alone. (C) OCl-AML3 cells were cultured alone or on MSC feeder layers followed by 6 hours of treatment with $100 \mu \mathrm{mol} / \mathrm{I} \mathrm{EX}$ alone or in combination with 1 or $3 \mu \mathrm{mol} / \mathrm{I}$ ABT-737. The levels of cytochrome $c$ in the cytosolic fraction were determined by immunoblotting. ( $\mathbf{D}$ and $\mathbf{E}$ ) OCl-AML3 cells were cultured as in $\mathbf{C}$ and exposed to $100 \mu \mathrm{mol} / \mathrm{l}$ EX for 6 hours. Mitochondrial suspensions were exposed to the indicated concentrations of ABT-737, and the release of AIF (D) and cytochrome $c(E)$ were determined by immunoblot. (F) MOLM-13 cells were cultured alone or on MSC feeder layers and exposed to 50 and $100 \mu \mathrm{mol} / \mathrm{I} \mathrm{EX}$ for 6 hours. Mitochondrial suspensions were exposed to $2 \mu \mathrm{mol} / \mathrm{l}$ $\mathrm{ABT}-737$, and the release of cytochrome $c$ and AIF were determined by immunoblot. may be more associated with cell survival than that in monocultures. Finally, the pan-caspase inhibitor z-VAD-fmk $(50 \mu \mathrm{M}) \mathrm{did}$ not diminish apoptosis induced by EX in MOLM13 cells (Supplemental Figure 7), with similar results in OCI-AML3 cells (data not shown). These results suggest that caspases are not required for the cytotoxic activity of this agent.

The cytotoxicity of FAO inbibition is independent of ROS or UCP2 activity. Because EX has been reported to induce ROS production (23), we investigated whether this agent promotes an increase in superoxide levels as measured by dihydroethidine oxidation. EX did not increase superoxide in OCI-AML3 or MOLM13 cells (Supplemental Figure 8), which suggests that the cytotoxic effects of EX is independent of ROS. Interestingly, although $\operatorname{EX}(50-100 \mu \mathrm{M})$ completely inhibited ${ }^{14} \mathrm{CO}_{2}$ generation from $\left[1-{ }^{14} \mathrm{C}\right]$-palmitate (data not shown), this agent did not promote marked accumulation of long-chain fatty acyl-CoA species (Supplemental Figure 9), presumably due to the reported inhibitory effect of EX on lipolysis (24), which may counteract an increase in free fatty acid pools. Nonetheless, this observation suggests that the growth-inhibitory effects of EX are not mediated by an increase in intracellular long-chain fatty acyl-CoAs. Lastly, because we recently reported that UCP2 and STAT3 are activated in leukemia cells cultured on MSC feeder layers $(2,25)$, we performed immunoblot analysis to determine whether EX modulates the expression of UCP2 and phosphorylation of STAT3 in OCI-AML3 and MOLM13 cells cultured alone or on MSC feeder layers. Our results revealed that in OCI-AML3 cells, EX dose-dependently decreased the expression of UCP2 in monocultures and inhibited STAT3 phosphorylation and increased UCP2 promoted by MSC feeder layers (Figure 2D). In contrast, in MOLM13 cells, EX increased the expression of UCP2 in monocultures and did not affect the expression of UCP2 or phosphorylation of STAT3 induced by MSC feeder layers (Figure 2D). Moreover, genetic ablation of UCP2 expression via siRNA methodology did not modulate EX cytotoxicity, and EX enhanced the cytotoxicity of the UCP2 inhibitor genipin (26) in MOLM13 cells, but not OCI-AML3 cells (data not shown). The above results suggest that the antileukemic effects of FAO inhibition are unrelated to the levels and/or activity of UCP2 or the phosphorylation of STAT3.

FAO inhibition sensitizes leukemia cells to apoptosis induction by ABT-737 and Nutlin $3 a$. Because metabolic stresses may promote cell death via activation of the proapoptotic $\mathrm{Bcl}-2$ protein $\operatorname{Bim}(27,28)$, we investigated whether inhibition of FAO modulates the expression of this protein in monocultures and MSC cocultures of leukemia cells. Intriguingly, as shown in Figure 2D, MSC coculture resulted 
A

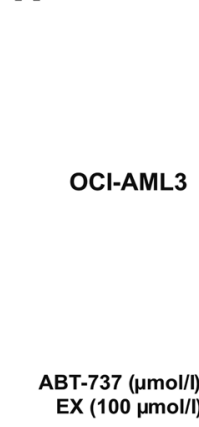

B
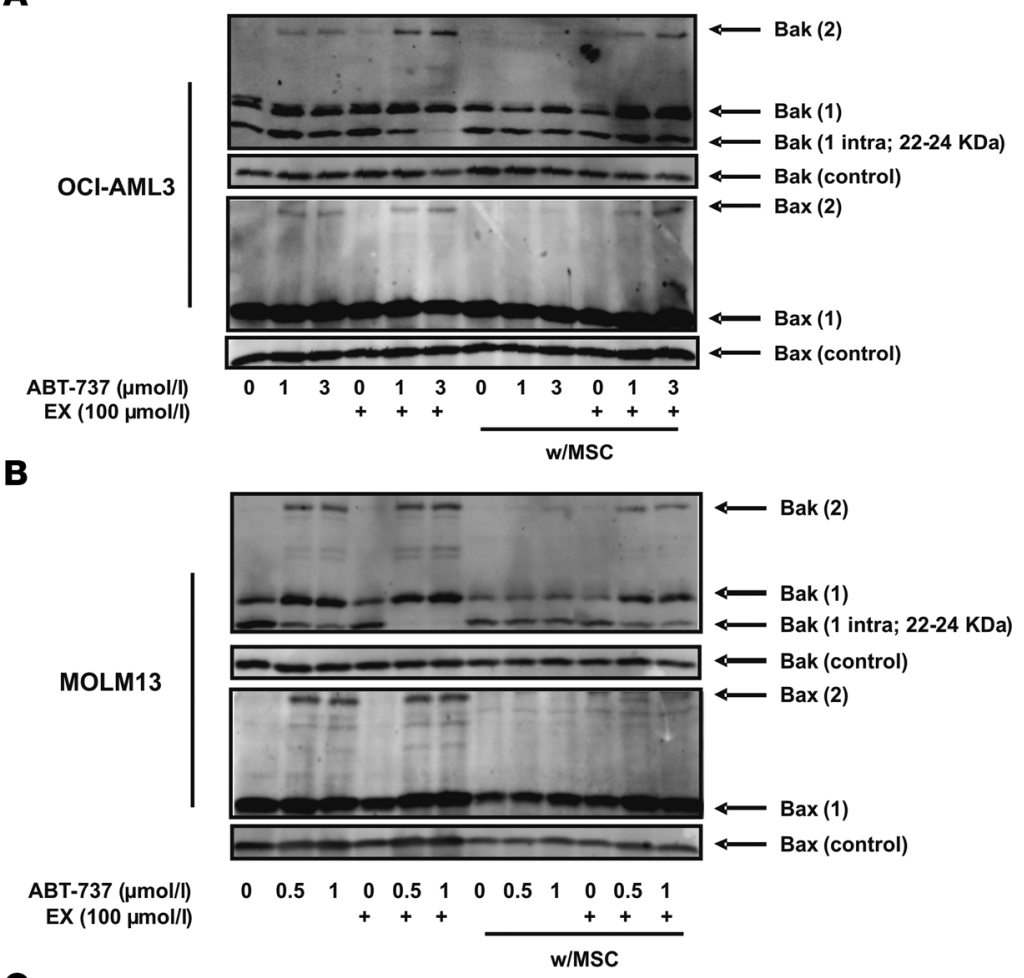

C

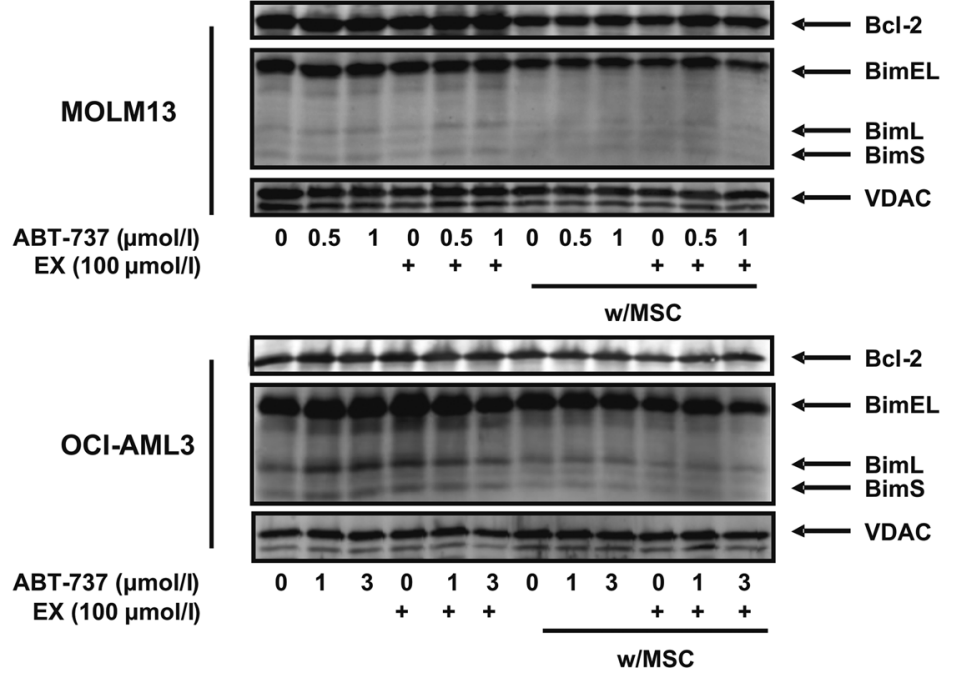

\section{Figure 5}

Inhibition of FAO facilitates Bak and Bax oligomerization. (A and $\mathbf{B}$ ) Monocultures and MSC cocultures of OCI-AML3 (A) and MOLM13 (B) cells were exposed to $100 \mu \mathrm{mol} / \mathrm{l} \mathrm{EX}$ alone or in combination with $\mathrm{ABT}-737$ ( 1 or $3 \mu \mathrm{mol} / \mathrm{l}$ for OCl-AML3; 0.5 or $1 \mu \mathrm{mol}$ for MOLM13) for 6 hours. Mitochondrial suspensions from leukemia monocultures and MSC cocultures (after MACS depletion of MSCs) were exposed to $0.4 \mathrm{mM}$ bismaleimidohexane and immunoblotted as described in Methods. The expression of Bax and Bak in untreated (uncrosslinked) mitochondrial lysates are shown as loading controls. (C) MOLM13 and OCl-AML3 cells were cultured and treated as in $\mathbf{A}$ and $\mathbf{B}$. Untreated (no bismaleimidohexane) mitochondrial fractions were immunoblotted for the indicated proteins. in decreased expression of the proapoptotic Bcl-2 family protein Bim, and this effect was partly antagonized by EX in a dose-dependent manner in MOLM13 cells, but not OCI-AML3 cells. Inhibition of FAO did not alter Bcl-2, Mcl-1, Puma, or Bax levels (data not shown). Because decreased expression of Bim may hinder activation of Bax and Bak and subsequent apoptosis (29), we investigated whether OCI-AML3 and MOLM13 cells cultured on MSC feeder layers would be resistant to apoptosis induction by ABT-737 and how $100 \mu \mathrm{mol} / 1 \mathrm{EX}$ modulated the response of leukemia cells to this $\mathrm{BH} 3$ mimetic. We used $100 \mu \mathrm{mol} / \mathrm{l}$ EX because this dose maximally inhibited oxygen consumption without inducing significant apoptosis at 48 hours. Additionally, because we and others have reported that increased p53 levels induce apoptosis via direct and indirect $\mathrm{Bcl}-2$ antagonism $(30,31)$, we similarly test- ed the interaction of EX with the MDM-2 antagonist Nutlin 3a under the same conditions. As shown in Figure 3A, OCI-AML3 and MOLM13 cells grown on MSC feeder layers were less sensitive to the proapoptotic effects of ABT-737, which supports the notion that decreased Bim expression and/or the increased FAO observed in coculture opposes the effects of BH3 mimetics. Nevertheless, EX sensitized both leukemia cell types, alone and in coculture, to apoptosis induction by ABT-737, suggesting that FAO per se may antagonize the proapoptotic effects of this agent. In contrast, MSC feeder layers did not significantly decrease apoptosis induction by Nutlin 3a in OCI-AML3 or MOLM13 cells (data not shown), although EX sensitized both cell types grown in monoculture to apoptosis induced by this agent (Figure 3B). The above observations suggest that in wild-type p53 cells, FAO inhibition may elicit 
A Spleen (IHC:GFP)

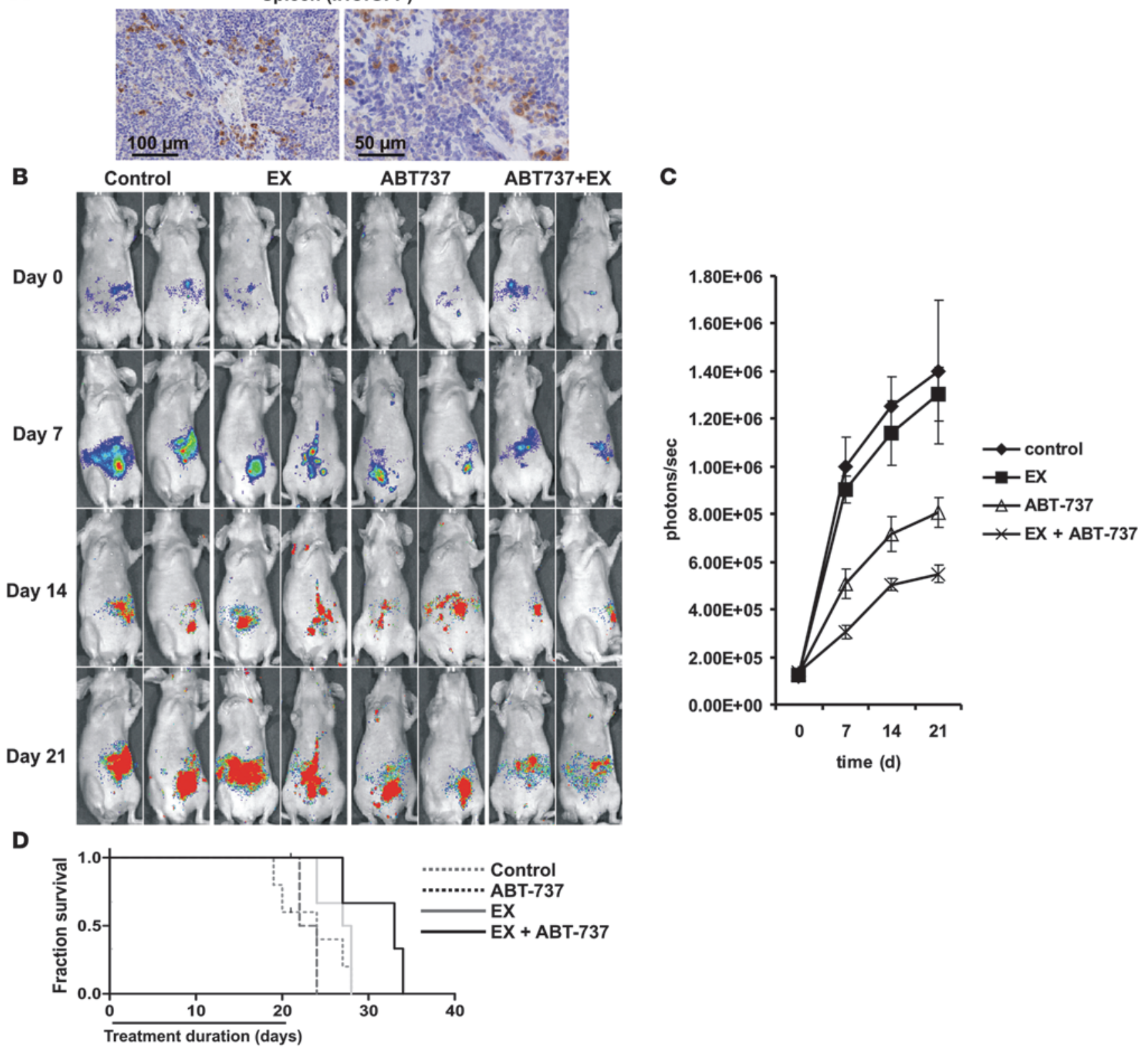

Figure 6

EX enhances the therapeutic efficacy of ABT-737 in a murine model of human AML. (A) At 5 weeks after i.v. injection of $2.5 \times 10^{6}$ GFP/luciferase-bearing MOLM13 cells, nude mice were sacrificed, and their spleens were analyzed by immunohistochemistry for GFP+ cells. Scale bars: $100 \mu \mathrm{m}$ (left); $50 \mu \mathrm{m}$ (right). (B and C) Nude mice xenotransplanted as in A were randomized and treated with control liposomes, ABT-737 liposomes, EX, or EX plus ABT-737, and leukemia burden was noninvasively analyzed as described in Methods. (D) Survival was estimated by Kaplan and Meier analysis as described in Methods. The EX plus ABT-737 treatment group was significantly different from the control $(P<0.005)$ and ABT-737 alone $(P<0.05)$ groups.

p53-dependent and -independent responses. Likewise, OCI-AML3 cells treated with ranolazine or siRNA targeting CPT1 were sensitized to apoptosis induction by ABT-737 and Nutlin 3a (Supplemental Figure 10 and Figure $3 \mathrm{C}$ ). Because our results suggest that in leukemia cells, fatty acid synthase/lipase inhibition by orlistat affects FAO, we investigated whether this agent could also sensitize leukemia cells to apoptosis induction by ABT-737. As shown in Figure 3D, orlistat sensitized OCI-AML3 cells - alone and in coculture with MSCs - to apoptosis induction by ABT-737, fur- ther supporting the notion that de novo synthesized and/or lipolysis-generated free fatty acids support survival in leukemia cells. Finally, although EX treatment did not increase p53 levels (data not shown), EX sensitized OCI-AML3 cells in which the expression of $\mathrm{p} 53$ was decreased by shRNA methodology (32) to ABT-737 (Figure 4A), which suggests that the proapoptotic effect of EX is independent of p53 activation. Similar sensitization to ABT-737 occurred in U937 cells, which carry a mutated p53 (Figure 4B). Taken together, the above results demonstrate, for the first time 
A

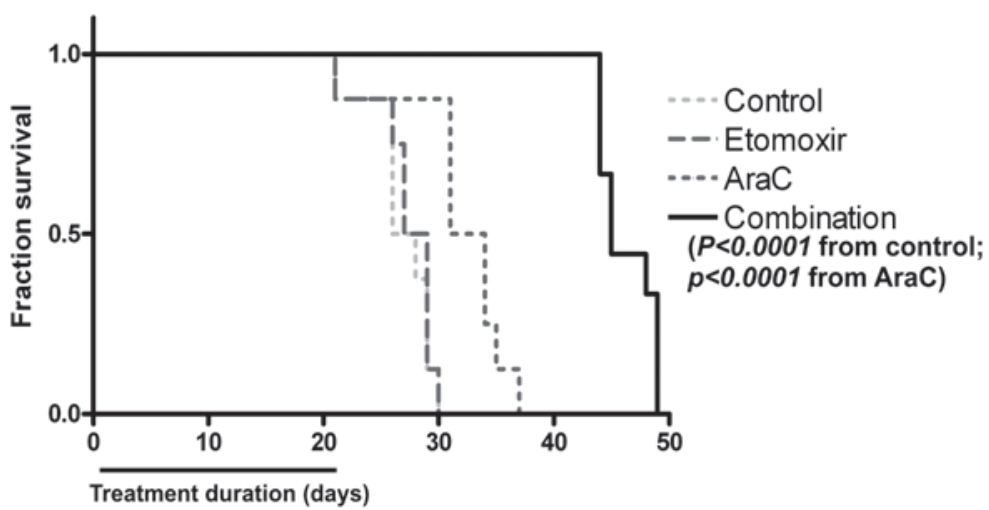

B

Control

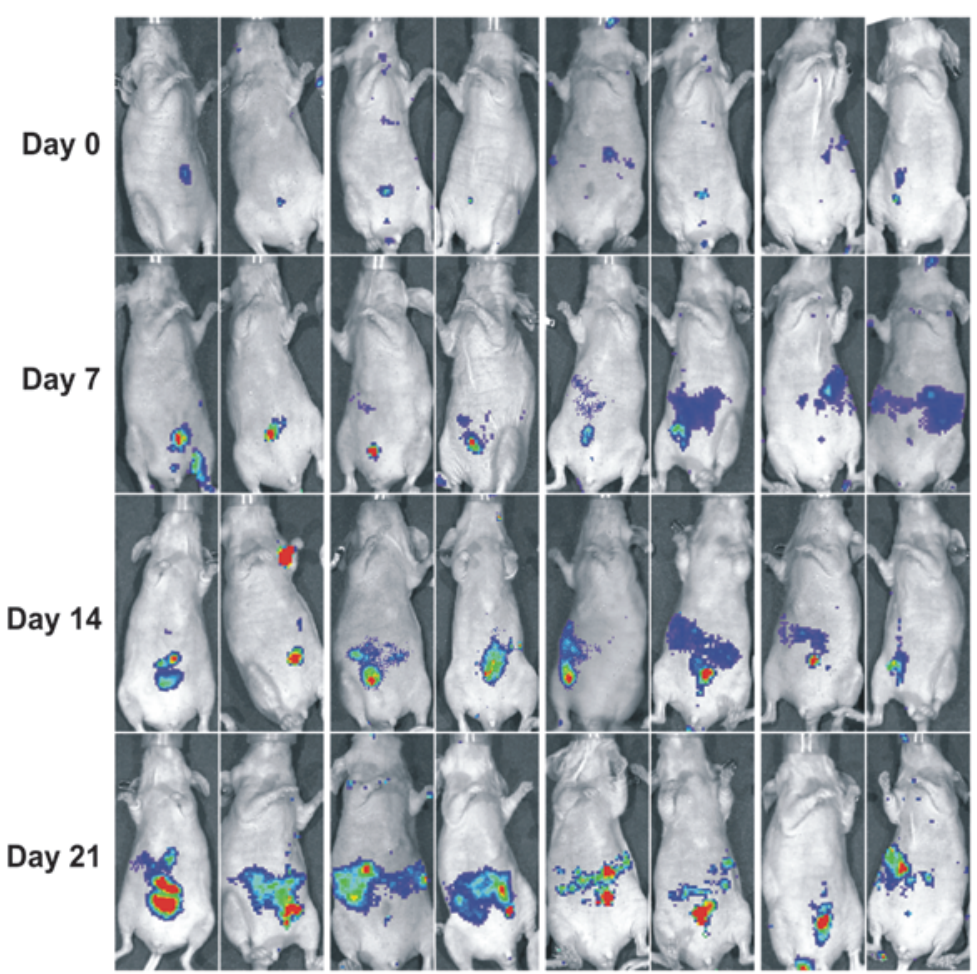

C

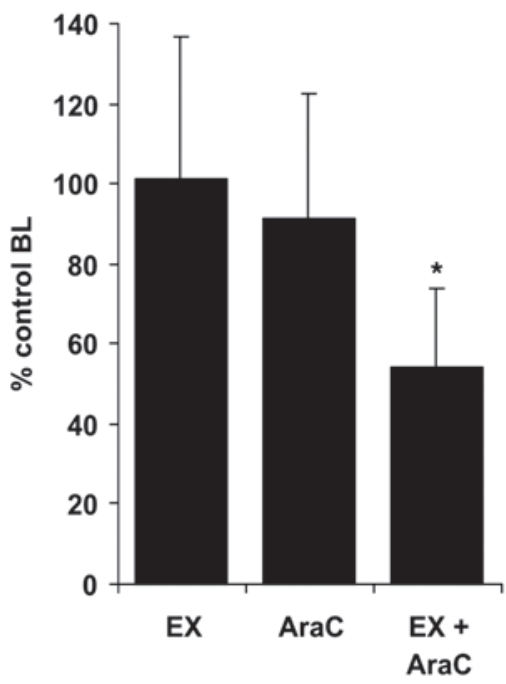

Figure 7

EX enhances the therapeutic efficacy of Ara-C in a murine model of human AML. (A) At 2 weeks after i.v. injection of $2.5 \times 10^{6}$ GFP/luciferase-bearing MOLM13 cells, nude mice were randomized and treated with EX, Ara-C (100 mg/kg i.p. every other day), or EX plus Ara-C for 3 weeks, and survival was estimated by Kaplan and Meier analysis as described in Methods. (B) Noninvasive imaging of leukemia burden and progression. (C) At 2 weeks after the start of treatment (4 weeks after xenotransplantation), leukemia burden was quantitated noninvasively via bioluminescence (BL) as described in Methods.

to our knowledge, that inhibition of FAO sensitizes leukemia cells to ABT-737 and Nutlin 3a and overcomes the protective effect of MSC feeder layers toward the $\mathrm{BH} 3$ mimetic.

Inbibition of FAO facilitates mitochondrial permeability transition after ABT-737 treatment. To further investigate the mechanism by which inhibition of FAO sensitizes leukemia cells to ABT-737induced apoptosis, we monitored the release of cytochrome $c$ in OCI-AML3 cells in monocultures and on MSC feeder layers after 6 hours of exposure to ABT-737 ( 1 and $3 \mu \mathrm{mol} / \mathrm{l}$ ), alone or in combination with $100 \mu \mathrm{mol} / \mathrm{l}$ EX. Figure 4C shows that MSC coculture opposed cytochrome $c$ release in response to ABT-737, and that EX sensitized OCI-AML3 cells (alone or in coculture) to the release of this apoptogenic factor, which suggests that FAO inhibition modulates the mitochondrial permeability transition pore (MPTP). Similar observations were made in monocultures of MOLM13 cells (data not shown). Next, to determine whether the sensitization effect of EX occurs via direct perturbations to the mitochondrial membrane, we isolated mitochondria from OCI-AML 3 cells treated with $100 \mu \mathrm{mol} / \mathrm{l} \mathrm{EX}$ and resuspended them in hyposmotic buffer, as described in Methods. The mitochondrial suspensions were then exposed to various doses of ABT-737, and the presence of apoptosis-inducing factor (AIF) and cytochrome $c$ 


\section{Table 1}

Leukemia patient sample characteristics

\begin{tabular}{|c|c|c|c|c|c|}
\hline Sample & Blast \% & Source & Diagnosis & FAB & Cytogenetics \\
\hline A & NA & BMA & AML & & Hyperdiploid metaphase 47,XY,+8[1]; Diploid male karyotype 46,XY[19] \\
\hline B & 55 & BMA & AML & M1 & $46, X Y[20]$ \\
\hline C & 77 & BMA & AML & M4 & Diploid female karyotype //46,XX[20] \\
\hline D & 70 & BMA & AML & & Not done \\
\hline $\mathrm{E}$ & 95 & BMA & AML & M0 & $46 X X[20]$ \\
\hline $\mathrm{F}$ & 30 & BMA & AML & M4 & Hyperdiploid clone $47, \mathrm{XY},+21 \mathrm{c}[20]$ \\
\hline G & 94 & PB & AML & M5B & $46, x x, \operatorname{add}(6)(p 23)[11] / 46, x x[9]$ \\
\hline $\mathrm{H}$ & 95 & PB & AML & M1 & $\begin{array}{l}47, \mathrm{XY},+11, \mathrm{t}(14 ; 19)(\mathrm{q} 11.2 ; \mathrm{q} 13.2)[12] ; 47, \mathrm{idem}, \mathrm{t}(1 ; 17)(\mathrm{p} 36.1 ; \mathrm{q} 21)[5] ; \\
47, \mathrm{XY}, \operatorname{del}(4)(\mathrm{q} 27 \mathrm{q} 31), \operatorname{add}(6)(\mathrm{p} 25), \operatorname{del}(6)(\mathrm{q} 21 \mathrm{q} 25),+11, \mathrm{t}(14 ; 19)(\mathrm{q} 11.2 ; \mathrm{q} 13.2)[1] ; 46, \mathrm{XY}[2]\end{array}$ \\
\hline I & 96 & BMA & ALL & & $47, X X,+X, t(4 ; 11)(q 21 ; q 23)[20]$ \\
\hline J & 79 & BMA & RAEB & & Not done \\
\hline K & NA & BMA & $\mathrm{CML}$ & $\mathrm{CH}$ & Pseudodiploid clone 46,XX,t(9;22)(q34;q11.2)[20] \\
\hline $\mathrm{L}$ & NA & BMA & CMML & & $46, X Y[20]$ \\
\hline M & NA & BMA & CMLA & & $47, X Y,+8[7] / 46, X Y[3]$ \\
\hline N & 89 & PB & AML & M5 & $46, X Y[20]$ \\
\hline 0 & 97 & PB & AML & & Diploid male karyotype 46,XY[20] \\
\hline$P$ & 48 & BMA & AML & M1 & Pending \\
\hline Q & 36 & PB & AML & M4 & Pending \\
\hline
\end{tabular}

FAB, French-American-British hematology classification; BMA, bone marrow aspirate; PB, peripheral blood; RAEB, refractory anemia with excess blasts. APh chromosome in this patient was not detected by routine cytogenetics. Diagnosis made by FISH LSI bcr/abl ES probe from Abbott Molecular Inc.

in the supernatant fraction was determined by immunoblot. As shown in Figure 4, D and E, mitochondria obtained from EX-treated OCI-AML3 cells were more susceptible to ABT-737-induced release of AIF and cytochrome $c$, which suggests that inhibition of FAO may directly sensitize mitochondria to the MPTP. Likewise, mitochondria derived from MOLM13 cells treated with 50 and $100 \mu \mathrm{mol} / 1 \mathrm{EX}$ - alone or from MSC cocultures - demonstrated increased sensitivity to ABT-737-induced AIF release (Figure 4F). Because ceramide can promote mitochondrial apoptosis (33), and because EX has been reported to increase the levels of ceramide (14), we hypothesized that an increase in ceramide may underlie the mitochondriotoxic effects of EX. However, ceramide content of OCI-AML3 and MOLM13 cells was not significantly altered after treatment with EX (Supplemental Figure 11). Nonetheless, these data support the notion that inhibition of FAO results in direct perturbations to the mitochondrial membrane that decrease the threshold for MPTP opening.

Inbibition of FAO facilitates Bak and Bax oligomerization. To investigate whether the observed facilitation of MPTP opening by inhibition of FAO is associated with Bax and Bak oligomerization, mitochondria obtained from OCI-AML3 and MOLM13 cells treated with $100 \mu \mathrm{mol} / \mathrm{l} \mathrm{EX}$ for 6 hours in the presence or absence of ABT-737 - alone or in coculture with MSCs - were exposed to the bifunctional crosslinking agent bismaleimidohexane. Immunoblot analysis for Bax and Bak demonstrated that MSC feeder layers inhibited the formation of Bax and Bak dimers after treatment with ABT-737 (Bax 2 and Bak 2, respectively; Figure 5, $A$ and $B$ ), indicating that the antagonism of apoptosis induced by this agent under coculture conditions may be related to decreased oligomerization of these proapoptotic Bcl-2 proteins. Additionally, treatment with EX promoted the ABT-737-dependent formation of Bak dimers, but not Bax dimers, in cells cultured alone, whereas this agent facilitated Bax and Bak dimer formation in cocultured cells treated with ABT-737. Interestingly, our observations also revealed that $\mathrm{FAO}$ inhibition in combination with ABT-737 pro- moted the exposure of the $\mathrm{N}$ terminus of Bak decreasing the intramolecular crosslinks between Cys14 and Cys166 that result in a Bak-immunoreactive band with a mobility of about 22-24 kDa (Bak 1 intra). A similar finding was observed by Ruffolo et al. when Bak activation was promoted by t-Bid (34), supporting the conclusion that exposure of the Bak $\mathrm{N}$ terminus is a critical step in promoting Bak oligomerization and apoptosis. Because Bim can activate Bak and induce its oligomerization, we investigated whether EX treatment, alone or in combination with ABT-737, increased Bim attachment to the mitochondrial membrane. As shown in Figure 5C, Bim expression was not altered under any condition in MOLM13 cells. In contrast, in OCI-AML3 mitochondria derived from monocultures, ABT-737 or EX - but not their combination - moderately increased the levels of Bim, although no changes in Bim expression were seen in mitochondria from OCI-AML3 cells grown on MSC feeder layers. This observation suggests that the observed decrease in Bim expression in whole cell extracts (Figure 2D) does not result in decreased expression of this proapoptotic protein in the mitochondrial fraction. No changes in the expression of $\mathrm{Bcl}-2$ were observed. These data suggest that sensitization to ABT-737 by FAO inhibition is likely not dependent on changes in the subcellular localization of Bim or Bcl-2; instead, EX may sensitize cells to MPTP opening via direct effect on Bak activation, which in turn may facilitate the observed oligomerization of Bax in leukemia cells cultured on MSC feeder layers.

In hibition of FAO enhances the therapeutic efficacy of ABT-737 and Ara-C in a murine model of human AML. To determine whether EX could potentiate the antileukemic effects of ABT-737 in vivo, we conducted an experiment in nude mice xenotransplanted with GFP/luciferase-bearing MOLM13 human leukemia cells. At 2 weeks after leukemia transplantation, mice were randomized and treated with liposomal ABT-737 (i.v. 20 mg/kg, every other day for 3 weeks), EX (i.p. $50 \mathrm{mg} / \mathrm{kg}$ every other day for 3 weeks), ABT-737 in combination with EX, or empty liposomes i.v. as a control. Engraftment of MOLM13 cells was evidenced by immunohistochemical 


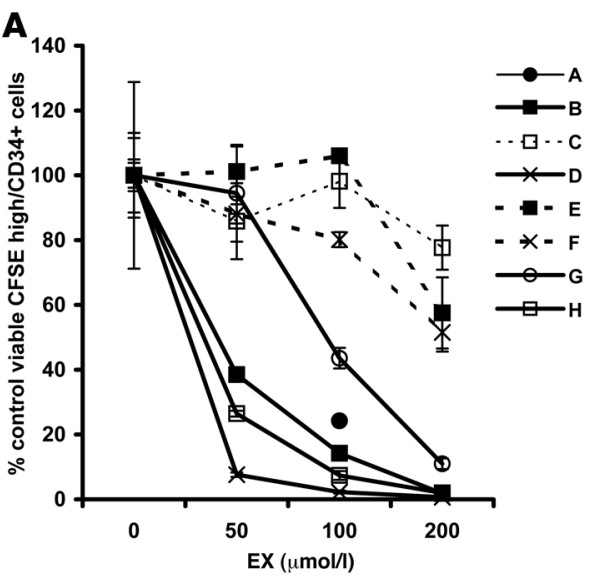

C

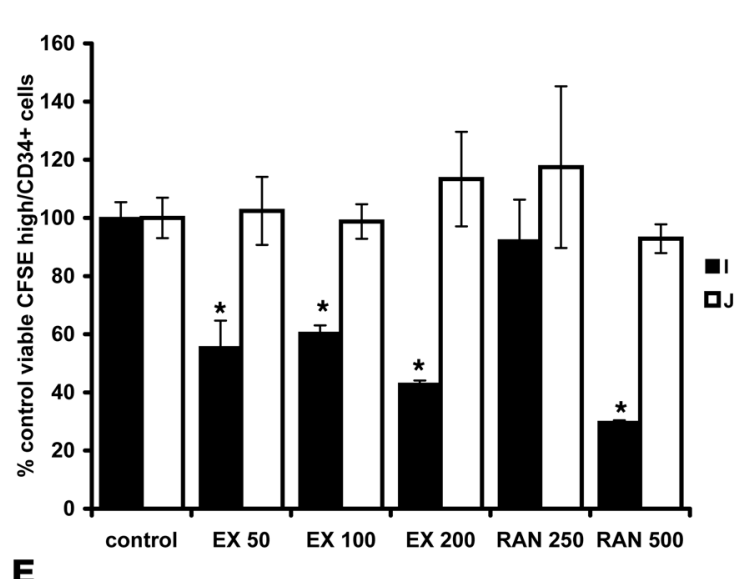

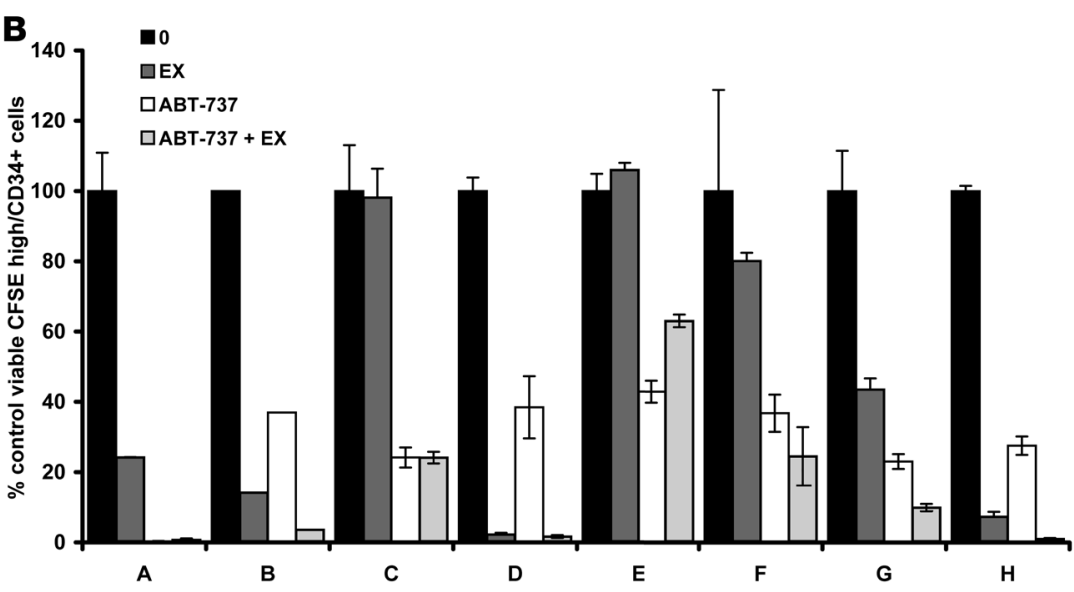

D
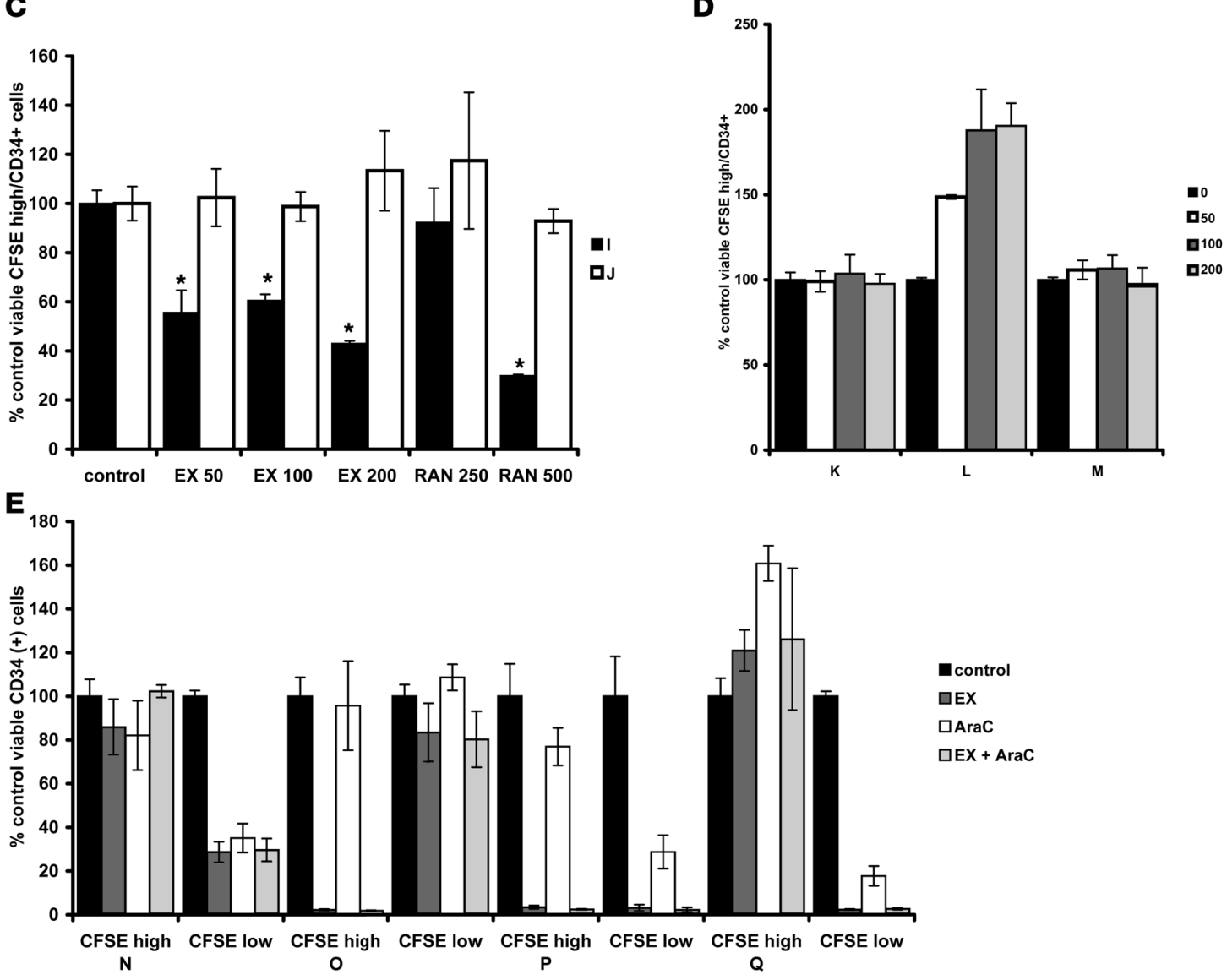

Figure 8

Inhibition of FAO can decrease the number of QLPs ex vivo. (A) Primary leukemia samples A-H were loaded with the cell tracing dye CFSE as described in Methods and exposed to increasing concentrations of EX for 5 days. Cells were then collected and stained with CD34-APC and 7-AAD, and viable CFSE ${ }^{\text {hi }} \mathrm{CD} 34^{+}$cells were quantitated by flow cytometry as described in Methods. Results show the mean \pm SD of 3 independent experiments, except for sample B, which shows results from duplicate experiments. Sample A was only treated with 100 umol/l EX. (B) At 20 hours prior to harvesting of samples A-H, $50 \mathrm{nmol} / \mathrm{l}$ ABT-737 was added to untreated cells or to cells exposed for 4 days to $100 \mu \mathrm{mol} / / \mathrm{EX}$. CFSEhiCD34+ cells were analyzed as in A. (C) Samples I and J were treated with EX or ranolazine (RAN) at the indicated doses (in $\mu \mathrm{mol} / \mathrm{l}$ ) for 5 days, and CFSEhiCD34+ cells were analyzed by flow cytometry. ${ }^{*} P<0.001$ versus control. (D) CML samples $\mathrm{K}$, L, and $\mathrm{M}$ were exposed to increasing concentrations of $\mathrm{EX}$ for 5 days and analyzed by flow cytometry gating on viable (samples $\mathrm{K}$ and $\mathrm{L}$ ) or viable and $\mathrm{CD}_{34}{ }^{+}$(sample M) cells. (E) AML samples were exposed to $100 \mu \mathrm{mol} / \mathrm{I} \mathrm{EX}$ alone or in combination with $100 \mathrm{nmol} / \mathrm{l} \mathrm{Ara}-\mathrm{C}$ for 5 days and analyzed by flow cytometry as in $\mathbf{A}$. 
detection of $\mathrm{GFP}^{+}$cells in the spleens of control mice 5 weeks after xenotransplantation (Figure 6A). Notably, although control and EX-treated mice demonstrated progressive increase in leukemiaderived bioluminescence, mice treated with ABT-737, and to a greater extent those treated with ABT-737 plus EX, appeared to resist tumor burden progression (Figure 6B). In addition, quantitation of leukemia-derived bioluminescence demonstrated that mice treated with ABT-737 plus EX had significantly lower leukemia burden than did control or ABT-737-treated mice $(P<0.00001$; Figure 6C). Most importantly, however, the combination of ABT-737 with EX significantly prolonged median survival by $33 \%$, from 24 days after start of treatment in the control group to 33 days $(P<0.005$, Log-rank [Mantel-Cox] test; Figure 6D). Neither ABT737 nor EX alone was able to significantly prolong median survival (ABT73, 27.5 days, $P=0.081$; EX, 23 days, $P=0.996$ ) compared with untreated control. At 33 days' median survival, ABT-737 plus EX was also significantly more efficacious than each agent alone (EX, 23 days; $P<0.001$; ABT-737, 27.5 days; $P<0.05$ ). Finally, although our in vitro results indicated that EX did not potentiate the cytotoxic effects of Ara-C (data not shown), we investigated whether EX could favorably interact with Ara-C in our murine model of leukemia. As shown in Figure 7A, the combination of EX with Ara-C significantly prolonged the median survival by $67 \%$ - from 27 days after start of treatment in the control group to 45 days ( $P<0.0001$, Log-rank [Mantel-Cox] test). Ara-C plus EX also provided a significant therapeutic benefit over Ara-C alone (Ara-C plus EX, 45 days; Ara-C, 32.5 days; $P<0.0001)$. Likewise, Ara-C plus EX was most effective in decreasing leukemia burden, as monitored by bioluminescence imaging $(P<0.0001$ versus Ara-C alone; Figure 7, B and $\mathrm{C}$ ). It bears mentioning that the cause of death of MOLM13-bearing mice treated with EX and ABT-737 or EX and Ara-C was most likely associated with disease progression (as evidenced by leukemia infiltration of liver and spleen; data not shown), leaving the following 2 possibilities: (a) that this regimen is not curative, or (b) that the 3-week treatment duration is insufficient. Nevertheless, the above results demonstrate that pharmacological inhibition of FAO in combination with ABT-737 or Ara-C synergistically decreases tumor burden and prolongs survival of nude mice engrafted with human leukemia suggesting the potential clinical utility of this regimen.

Inbibition of FAO can decrease the number of QLPs ex vivo. QLPs are of ominous importance in AML therapy because in most primary samples, these cells have the ability to initiate leukemia in NOD/ SCID mouse models (35). Moreover, we have observed that these cells are resistant to traditional chemotherapeutic agents used in AML treatment (36). A priori we hypothesized that EX and ranolazine should target highly proliferative cells over QLP cells, because our in vitro models are representative of highly proliferative cells that may rely in large part on increased Krebs cycle activity and continuous oxygen reduction for survival. To test this hypothesis, we collected samples from patients with primary AML and chronic myelogenous leukemia (CML) via peripheral blood draws and bone marrow aspirates and loaded them with the cell tracing reagent CFSE. Patient characteristics are listed in Table 1; note that sample I, initially diagnosed as CML, was rediagnosed as acute lymphoblastic leukemia (ALL). CFSE is cell permeable; upon removal of the acetate moieties by intracellular esterases, this agent reacts with intracellular amines, forming stable, fluorescent adducts that decrease proportionally to cell division, allowing the flow cytometric identification of quiescent/slowly proliferating cell populations (CFSE ${ }^{\text {hi }}$ ). After staining with CFSE, samples were cultured for 5 days alone or in the presence of increasing concentrations of EX or ranolazine; in some samples, ABT-737 was added 20 hours prior to harvest. At the end of the experiment, absolute numbers of viable $\mathrm{CFSE}{ }^{\text {hi }} \mathrm{CD} 34^{+}$cells were quantitated by flow cytometry. As shown in Figure 8A, 3 of 8 samples demonstrated decreases in CFSE ${ }^{\text {hi }} \mathrm{CD} 34^{+}$cells with $50 \mu \mathrm{mol} / 1 \mathrm{EX}$ treatment, 5 of 8 demonstrated decreased CFSEhiCD34+ ${ }^{+}$cells with $100 \mu \mathrm{mol} / 1$ EX treatment, and 1 sample did not respond to treatment with $200 \mu \mathrm{mol} / 1$ of this agent. Supplemental Figure 12 shows representative histograms of CFSE intensity gated on viable CD $34^{+}$cells: depending on the sample, EX decreased quiescent cells (sample H); decreased quiescent and proliferating cells (sample G); decreased proliferating, but not quiescent, cells (samples J and C); or failed to target either quiescent or proliferating cells (sample E). We did not observe an increase in the number of proliferating cells after treatment with EX in any sample examined (data not shown). Treatment with $50 \mathrm{nmol} / 1 \mathrm{ABT}-737$ for 20 hours prior to harvest was very effective in decreasing $\mathrm{CFSE}^{\mathrm{hi}} \mathrm{CD} 34^{+}$cells in all samples (Figure $8 \mathrm{~B}$ ). In 5 of 8 samples, the combination of $100 \mu \mathrm{mol} / \mathrm{l} \mathrm{EX}$ and ABT-737 was more effective than each agent alone (Figure $8 \mathrm{~B}$ ). Additionally, in a separate experiment, we observed that ranolazine also decreased the number of viable $\mathrm{CFSE}^{\text {hi }} \mathrm{CD} 34^{+}$cells in $1 \mathrm{ALL}$ sample that was sensitive to EX (sample I; Figure 8C), although this agent was ineffective in 1 refractory anemia with excess blasts sample that was also resistant to EX (sample J; Figure 8C), which supports the notion that both agents induce cytotoxicity in quiescent cells via a similar mechanism. Of note, EX failed to decrease the number of quiescent cells in 2 CML samples (samples $\mathrm{K}$ and $\mathrm{M})$ and 1 chronic myelomonocytic leukemia (CMML) sample (sample L) investigated (Figure 8D); in sample L, this agent actually increased the number of CFSE ${ }^{\text {hi }}$ cells, which suggests that FAO inhibition in certain CML samples may block the progression from quiescence to proliferation (see Supplemental Figure 13 for the CFSE histogram of sample L). Because the mechanisms by which EX enhances Ara-C efficacy in vivo remain elusive, we also investigated whether the combination of these 2 agents targets $\mathrm{CFSE}^{\text {hi CD34 }}{ }^{+}$cells in AML. As shown in Figure 8E, CFSE ${ }^{\text {hi }} \mathrm{CD} 34^{+}$ cells from all AML samples examined were resistant to the cytotoxic effects of Ara-C, and 2 samples in which CFSEhi CD $34^{+}$cells were resistant to the cytotoxic effects of EX (samples N and Q) remained resistant to the combination of Ara-C and EX. In contrast, only 1 sample contained $\mathrm{CFSE}^{\mathrm{lo}} \mathrm{CD} 34^{+}$cells resistant to the cytotoxic effects of Ara-C, and EX did not overcome this phenotype. Finally, we observed that EX also decreased the number of viable normal quiescent $\mathrm{CD}_{3} 4^{+}$progenitors ex vivo (Supplemental Figure 14), which needs to be further investigated. Taken together, the above results suggest that FAO inhibitors have the potential to target QLP cells in AML, although the mechanisms for this effect remain to be elucidated.

\section{Discussion}

In a review published in 1956, Otto Warburg advanced the hypothesis that the respiration of cancer cells was damaged, resulting in a proglycolytic phenotype in the presence of oxygen (1). The abolition of the Pasteur effect (the inhibition of lactate generation in the presence of oxygen) in tumors became known as the Warburg effect. However, for several decades, the search for permanent, transmissible injuries to mitochondrial respiration that could support Warburg's hypothesis has not yielded any convincing results. 
Interestingly, recent observations suggest that in leukemia cells, the Warburg effect can be orchestrated not by mitochondrial damage per se, but instead by increasing the proton conductance of mitochondria, essentially uncoupling the synthesis of ATP from electron transport and oxygen consumption (2). In addition, high rates of aerobic glycolysis can occur independently of mitochondrial dysfunction (reviewed in ref. 37). Notably, mitochondrial uncoupling is characterized by decreased entry of pyruvate into the Krebs cycle in the presence of persistent oxygen consumption, possibly suggesting a shift to the oxidation of other carbon sources (38). Moreover, mitochondrial uncoupling has been demonstrated to promote FAO (39); conversely, FAO has been shown to induce mitochondrial uncoupling (40), at least in part via feed-forward activation of PPAR $\alpha$-regulated UCP3 (41). It is thus tempting to speculate that mitochondrial uncoupling in leukemia cells may represent a shift to unregulated FAO.

Here we present evidence to suggest that FAO largely supports oxygen consumption in leukemia cells and that this process is uncoupled from oxidative phosphorylation. This constrains leukemia cells to glucose metabolism for their energy needs. Of note, this metabolic constraint for the generation of ATP has contributed to the success of antiglycolytic agents as cancer chemotherapeutics (reviewed in ref. 42). Our results also suggest that MSC feeder layers augment this metabolic pattern, at least in part via increased dependence on de novo FAS, as well as by the previously reported activation of UCP2 expression (2). Interestingly, pharmacological FAO inhibitors, which promote glucose oxidation in the heart (20), did not promote pyruvate oxidation in leukemia cells. Instead, these inhibitors increased the amount of lactate generated by leukemia cells. Because pyruvate may serve as an anaplerotic substrate to support the oxidation of fatty acid-derived acetyl-CoA (9), inhibition of FAO may also decrease anaplerotic flux through pyruvate carboxylation. In addition, it is likely that the apparent increase in glycolytic activity induced by EX is an adaptive mechanism to maintain ATP production in the face of reduced PDH activity (Supplemental Figure 3). Nevertheless, our results suggest that leukemia cells are prone to oxidize fatty acids, but not pyruvate, via mitochondrial pathways.

Our results also demonstrated that pharmacological or genetic means of abolishing FAO sensitized leukemia cells to the proapoptotic effects of agents that directly activate the intrinsic apoptotic pathway, namely ABT-737 and Nutlin 3a. By extension of our metabolic observations, a similar sensitizing effect was observed in cells treated with the fatty acid synthase/lipase inhibitor orlistat, which supports the notion that de novo synthesized and/or lipolysis-generated free fatty acids are essential to support leukemia cell survival. Mechanistically, this was associated with a sensitization effect toward MPTP opening, because mitochondria isolated from cells treated with EX were more prone to the release of AIF and cytochrome $c$. It is important to point out that although leukemia cells grown on MSC feeder layers demonstrated reduced levels of Bim expression in whole cell extracts, we did not detect significant differences in Bim levels at the mitochondrial level before or after treatment with EX. Moreover, our results suggest that the facilitation of MPTP opening was promoted by increased exposure of the $\mathrm{N}$ terminus and dimerization of Bak, supporting a model in which inhibition of FAO results in perturbations to the mitochondrial membrane that result in enhanced activation of Bak by Bim and/or decreased sequestration of Bak by $\mathrm{Bcl}-2$ or $\mathrm{Mcl}-1$ (43). Although direct perturbations to the mitochondrial membrane by inhibition of FAO - via accumulation of ceramide $(14,33)$ or free fatty acids $(44)-$ could be a proposed mechanism for the observed effects, our data suggest that EX does not lead to ceramide or long-chain fatty acyl-CoA accumulation in leukemia cells. Alternatively, it is conceivable that inhibition of mitochondrial FAO may result in increased generation of toxic dicarboxylic acids via microsomal $\omega$-oxidation of excess fatty acids (45). Nevertheless, the synergism between orlistat (which does not promote ceramide or palmitate accumulation) and ABT-737 or Nutlin 3a motivates us to contemplate the possibility that fatty acid entry and/or FAO in mitochondria may ipso facto be involved in the regulation of the Bcl-2 apoptotic rheostat in leukemia cells. This last notion is supported by the previous observations that CPT- 1 interacts with t-Bid (16) and with Bcl-2 (17), although the contributions of CPT-1 activity to $\mathrm{t}$-Bid-induced apoptosis remain to be determined. Additional experiments will be required to address the significance of these interactions in the activation of the MPTP.

Our observations also demonstrated that inhibition of FAO had a marked antiproliferative effect - associated in part with apoptosis induction - in leukemia cells. Although the mechanisms orchestrating this phenomenon remain to be investigated, it appears unlikely to be mediated by p53 activation or ceramide accumulation, potentially suggesting instead that reduced Krebs cycle flux or decreased intramitochondrial NADH pools are contributing factors (46). Curiously, both EX and ranolazine decreased QLPs in approximately $50 \%$ of AML samples, which suggests that FAO may support the maintenance of these leukemia-initiating cells. The therapeutic relevance of these in vitro effects is not obvious in our in vivo leukemia model, in which EX alone had no significant effect on leukemia burden or survival. Additionally, the mechanism by which EX and Ara-C provided a therapeutic effect in vivo without demonstrating synergy in vitro is still unresolved. Nevertheless, our observations that genetic or pharmacological inhibition of FAO sensitized leukemia cells to ABT-737 and Nutlin 3a, and that EX provided a therapeutic benefit in a murine model of human leukemia in combination with ABT-737 or Ara-C, generate proof of principle that $\mathrm{FAO}$ can be a bona fide target for sensitizing hematological malignancies to agents that activate the intrinsic apoptotic pathway.

In conclusion, our results lead to 2 hypotheses. The first is that leukemia cells oxidize fatty acids. Uncoupling of oxidative phosphorylation promotes a shift of ATP production from FAO to glycolysis. Second, our data support the notion that this metabolic adaptation in leukemias is fundamentally linked to the Bcl-2 apoptotic rheostat and can be targeted for therapeutic intervention. Although the precise mechanism by which FAO inhibitors provide a therapeutic benefit in combination with ABT-737 or Ara-C in murine models of leukemia remain to be elucidated, we propose that modulation of fatty acid metabolism may represent a novel strategy for the treatment of hematological malignancies.

\section{Methods}

Primary leukemia samples. Bone marrow or peripheral blood samples were obtained for in vitro studies from patients with AML or CML. Samples were collected during routine diagnostic procedures after informed consent was obtained; protocols for studies in humans were approved by the Human Subjects Committee of the University of Texas M.D. Anderson Cancer Center. Mononuclear cells were separated by Ficoll-Hypaque (Sigma-Aldrich) density gradient centrifugation. 
Murine leukemia model. All studies in mice were reviewed and approved by the University of Texas M.D. Anderson Cancer Center IACUC. Via tail vein injection, we transplanted 5-week-old 01B74 athymic nude $(n u / n u)$ mice (NCI) with $2 \times 10^{6}$ MOLM13 cells stably expressing a dual Renilla luciferase-GFP reporter. At 2 weeks after xenotransplantation, mice were randomized into 4 treatment groups of 9 mice per group and treated as follows: liposomal ABT-737 (20 mg/kg i.v. every other day for 3 weeks), EX (50 mg/kg i.p. every other day for 3 weeks), ABT-737 plus EX, or empty liposomes as a control. In a separate experiment, xenotransplanted mice were randomized into 4 treatment groups of 8 mice per group and treated as follows: Ara-C (100 mg/kg i.p. every other day for 3 weeks), EX $(50 \mathrm{mg} / \mathrm{kg}$ i.p. every other day for 3 weeks), Ara-C plus EX, or untreated control. Leukemia burden was monitored by noninvasive imaging of isoflurane-anesthetized mice injected i.p. with luciferin in the In vivo Imaging System (Xenogen/Caliper Life Sciences), with total imaging time of 1 minute. Mice were sacrificed when they became moribund or unable to obtain food or water according to IACUC policies. In addition, 3 randomly assigned mice per group were sacrificed on day 35 after xenotransplantation for assessment of engraftment by GFP immunohistochemical staining. Survival was estimated with the product-limit estimator of Kaplan and Meier, and the log-rank statistic was used to test for differences in survival distributions between groups. To verify engraftment of MOLM13 cells, mice were randomly chosen from the control groups and sacrificed, and the presence of MOLM13 cells in the liver and spleen was assessed by immunohistochemistry.

Flow cytometric determination of $C F S E^{\text {hi }} \mathrm{CD} 34^{+}$lenkemia progenitors. Freshly isolated peripheral blood or bone marrow samples from leukemia patients were washed in PBS and resuspended in serum-free RPMI containing $1 \mu \mathrm{mol} / 1$ CFSE (Invitrogen). Samples were incubated for 10 minutes at $37^{\circ} \mathrm{C}$, washed twice in RPMI supplemented with $10 \%$ fetal calf serum, and resuspended at a cell density of $1 \times 10^{6} \mathrm{cells} / \mathrm{ml}$. As a control for quiescent cells, samples were treated with colcemid (100 ng/ml; Invitrogen). After treatments, cells were resuspended in $100 \mu \mathrm{l}$ Annexin binding buffer $(140 \mathrm{mM} \mathrm{NaCl}, 10 \mathrm{mM}$ $\mathrm{KH}_{2} \mathrm{PO}_{4}$, and $5 \mathrm{mMCaCl}_{2}, \mathrm{pH} 7.4$ ) containing $5 \mu \mathrm{g} / \mathrm{ml}$-amino actinomycin D (7-AAD; Sigma Aldrich), a 1:100 dilution of CD34-APC (BD Biosciences), and 20,000 CountBright flow cytometry counting beads (Invitrogen). After 15 minutes of incubation at room temperature, samples were analyzed by flow cytometry gating on live cells by forward and side scatter as well as 7-AAD negativity. Absolute numbers of CFSEhi (as determined by CFSE signal from colcemid control) and $\mathrm{CD} 34^{+}$cells are reported.

Cell lines, chemicals, and biochemicals. OCI-AML3, MOLM13, HL60, U937, OCI-AML3 vector shRNA, and OCI-AML3 p 53 shRNA cells were maintained in RPMI supplemented with $5 \%$ fetal calf serum, $1 \%$ glutamine, $100 \mathrm{U} / \mathrm{ml}$ penicillin, and $100 \mu \mathrm{g} / \mathrm{ml}$ streptomycin in a $37^{\circ} \mathrm{C}$ incubator containing $5 \%$ $\mathrm{CO}_{2}$. OCI-AML3 vector shRNA and OCI-AML3 p53 shRNA are stable clones of the OCI-AML3 cells that carry an empty shRNA-expressing vector and the same vector expressing a p53-targeted shRNA, respectively (32). EX and ranolazine were obtained from Sigma-Aldrich and dissolved in water. ABT-737 was synthesized at University of Texas M.D. Anderson Cancer Center based on the previously published structure (47) and dissolved in DMSO.

Lenkemia-stroma coculture. MSCs were derived from normal bone marrow samples obtained with informed consent in accordance with regulations and protocols approved by the Human Subjects Committee of the University of Texas M.D. Anderson Cancer Center. MSCs were cultured at a density of $1-5 \times 10^{4}$ cells $/ \mathrm{cm}^{2}$ in Mesenpro medium (Invitrogen), then seeded as feeder layers at $1.5 \times 10^{4}$ cells $/ 1.9 \mathrm{~cm}^{2}$ in 24 -well plates or T-75 flasks in RPMI medium 16 hours before addition of $2 \times 10^{5}$ cells $/ \mathrm{ml}$ (for 48 - to 96-hour experiments) or $5 \times 10^{5}$ cells $/ \mathrm{ml}$ (for all other experiments) MOLM13 or OCI-AML3 cells, or $1 \times 10^{6}$ primary leukemia cells $/ \mathrm{ml}$, in $1 \mathrm{ml}$ fresh RPMI medium. Cocultures were incubated for an additional
24-48 hours, nonadherent leukemia cells were removed, and fresh RPMI medium was replaced. The number of leukemia cells attached to MSCs was quantitated by flow cytometry using CountBright beads following the manufacturer's instructions (Invitrogen), and control cultures of leukemia cells alone were seeded in replicate plates or flasks at the same density. MSCs were depleted from cocultures by MACS separation using anti-APC microbeads (Miltenyi Biotech) after CD90-APC immunostaining.

Measurement of lactate generation, oxygen consumption, and ATP levels. Lactate levels and polarographic measurements of oxygen consumption were carried out as previously described (48). Fluorometric oxygen measurements using BD Oxygen Biosensor plates (BD Biosciences) were carried out as previously described (49). ATP levels were quantitated using the ATP bioluminescence kit CLS II (Roche Applied Science) according to the manufacturer's instructions.

Measurement of apoptosis and viable cell numbers by flow cytometry. After appropriate treatments, cells were washed twice in PBS and then resuspended in $100 \mu \mathrm{l}$ Annexin binding buffer containing a 1:100 dilution of Annexin V-FLUOS (Roche Applied Science) and $50 \mathrm{nmol} / 1$ tetramethyl-rhodamine methyl ester; where appropriate for MSC coculture experiments, a 1:100 dilution of anti-CD90 APC-conjugated antibody was added. CD90 was used to discriminate MSCs (positive) from leukemia cells (negative). In some experiments, cell numbers were quantitated after the addition of 10,000 CountBright counting beads (Invitrogen) per sample. Cells were then analyzed by flow cytometry in a FACSCalibur flow cytometer (BD Biosciences) using a 488-nm argon ion and 633-nm $\mathrm{HeNe}$ excitation lasers.

Mitochondrial isolation, cytochrome cand AIFrelease, and Bax and Bak crosslinking. After appropriate treatments and MACS separation, OCI-AML3 and MOLM13 cells were washed in 10 volumes of ice cold PBS and centrifuged. Mitochondria were isolated as previously described (48). For cytochrome $c$ and AIF release, mitochondria were resuspended in M buffer $(125 \mathrm{mM}$ $\mathrm{KCl}, 20 \mathrm{mM}$ HEPES, $10 \mathrm{mM}$ Tris-Cl, and $2 \mathrm{mM} \mathrm{KPO}_{4}$, $\mathrm{pH}$ 7.2) at $1 \mathrm{mg} / \mathrm{ml}$ protein and equilibrated at room temperature for 2 minutes prior to the addition of ABT-737. The concentration of DMSO in the solution did not exceed $0.2 \%$. Mitochondrial suspensions were incubated for 15 minutes at room temperature, and mitochondria were collected by centrifugation at $11,000 \mathrm{~g}$ for 5 minutes. The presence of cytochrome $c$ was evaluated by Western blotting of the mitochondrial pellet and the supernatant. Bax and Bak crosslinks were investigated as previously described (34). Briefly, mitochondria were resuspended in $150 \mathrm{mM} \mathrm{NaCl}, 10 \mathrm{mM}$ HEPES ( $\mathrm{pH}$ 7.4), and $1 \%$ CHAPS at $1 \mathrm{mg} / \mathrm{ml}$ of protein and treated with $0.4 \mathrm{mM}$ bismaleimidohexane (Thermo Scientific) for 1 hour at room temperature. We immunoblotted $12.5 \mu \mathrm{g}$ of protein for Bax and Bak.

Western blot analysis. Rabbit anti-Bim and mouse anti-Bak antibodies were purchased from Calbiochem. Mouse anti-Noxa and goat anti-CPT1 antibodies were obtained from Abcam. Rabbit anti-UCP2 and rabbit antiVDAC antibodies were purchased from Millipore, and rabbit anti-Bax and mouse anti-cytochrome $c$ antibodies were obtained from BD Biosciences. Goat anti-AIF antibody was obtained from Santa Cruz Biotechnology Inc. After appropriate treatments, cell extracts were generated and immunoblotted as previously described (48).

siRNA transfection. Silencing of CPT1 gene expression in leukemic cells was achieved by the siRNA technique. siGENOME SMART pool human CPT1 (liver isoform) siRNAs were obtained from Dharmacon. A nonspecific control pool, containing 4 pooled nonspecific siRNA duplexes, was used as a negative control. Transfection of leukemic cells was carried out by electroporation using the Nucleofection system (Amaxa) as previously described (18).

Cell extraction and ${ }^{13} \mathrm{C}-\mathrm{NMR}$ analysis. OCI-AML 3 cells were cultured alone or in MSC feeder layers in the presence of $11 \mathrm{mmol} / 1\left[1-{ }^{13} \mathrm{C}\right]$ glucose for 48 hours. Subsequently, $2 \times 10^{7}$ OCI-AML3 cells from cocultures (after MACS 
separation) and from single culture were centrifuged and rinsed with icecold saline. Cells were fixed in $10 \mathrm{ml}$ ice-cold methanol with constant vortexing, followed by the sequential addition of $10 \mathrm{ml}$ ice-cold chloroform and $10 \mathrm{ml}$ ice-cold deionized water. After phase separation and solvent removal, the lipid fraction was reconstituted in deuterated chloroform (lipid phase). ${ }^{13} \mathrm{C}$ spectra were acquired as previously described (50). A representative spectra from 3 independent experiments is shown.

Measurement of ceramides, long-chain fatty acyl CoA, and oleate oxidation. See Supplemental Methods.

Statistics. Unless otherwise indicated, results are expressed as mean \pm SD of 3 independent experiments. For immunoblot analyses, a representative immunoblot from 4 independent experiments is shown. $P$ values were determined by 1 -way ANOVA followed by $F$ statistics. A $P$ value less than 0.05 was considered significant.

\section{Acknowledgments}

We are grateful to Vivian Ruvolo and Paul Corn for the generation of lentivirally transduced OCI-AML3 cells and to Victor Lavis, Peng Huang, and Mong Hong Lee for stimulating discussions. We thank Patrick Guthrie for his invaluable help with FAO assays, and Wendy Schober and Karen Clise-Dwyer for their assistance with flow cytometry. We also acknowledge Leslie Calvert for laboratory assistance and Bradley Tadlock for administrative assistance. The present work was supported in part by a Young Investigator Award from LeukemiaTexas (to I. Samudio) and the Paul and Mary Haas Chair in Genetics (to M. Andreeff) as well as by NIH grants from the National Cancer Institute (P01-CA55164, P01-CA16672, and P01-CA49639, to $\mathrm{M}$. Andreeff) and from the National Heart, Lung, and Blood Institute (R01-HL-73162, to H. Taegtmeyer).

Received for publication February 17, 2009, and accepted in revised form October 21, 2009.

Address correspondence to: Michael Andreeff, University of Texas M.D. Anderson Cancer Center, 1515 Holcombe Boulevard, Unit 448, Houston, Texas 77030, USA. Phone: (713) 792-7260; Fax: (713) 794-4747; E-mail: mandreef@mdanderson.org. Or to: Heinrich Taegtmeyer, University of Texas Houston Medical School, 6431 Fannin Street, MSB 1.220, Houston, Texas 77030, USA. Phone: (713) 500-6569; Fax: (713) 500-0637; E-mail: Heinrich. Taegtmeyer@uth.tmc.edu.

Ismael Samudio's present address is: Department of Nutrition and Biochemistry, Pontificia Universidad Javeriana, Bogotá, DC, Colombia.
1. Warburg O. On the origin of cancer cells. Science. 1956;123(3191):309-314

2. Samudio I, Fiegl M, McQueen T, Clise-Dwyer $\mathrm{K}$, Andreeff M. The Warburg effect in leukemiastroma cocultures is mediated by mitochondrial uncoupling associated with uncoupling protein 2 activation. Cancer Res. 2008;68(13):5198-5205.

3. Dazzi F, Ramasamy R, Glennie S, Jones SP, Roberts I. The role of mesenchymal stem cells in haemopoiesis. Blood Rev. 2006;20(3):161-171.

4. Ho $\mathrm{AD}$, Wagner W. Bone marrow niche and leukemia. Ernst Schering Found Symp Proc. 2006;(5):125-139.

5. Konopleva M, Andreeff M. Targeting the leukemia microenvironment. Curr Drug Targets. 2007;8(6):685-701

6. Lynen F. Die Rolle der Phosphorsaeure bei Dehydrierungsvorgaengen und ihre biologische Bedeutung. Naturwiss. 1951;30:398-406.

7. Befroy DE, Petersen KF, Dufour S, Mason GF, Rothman DL, Shulman GI. Increased substrate oxidation and mitochondrial uncoupling in skeletal muscle of endurance-trained individuals. Proc Natl Acad Sci U S A. 2008;105(43):16701-16706.

8. Harper ME, et al. Characterization of a novel metabolic strategy used by drug-resistant tumor cells. FASEB J. 2002;16(12):1550-1557.

9. Goodwin GW, Taegtmeyer H. Improved energy homeostasis of the heart in the metabolic state of exercise. Am J Physiol Heart Circ Physiol. 2000;279(4):H1490-H1501.

10. Rosenfeld G. Fett und Kohlenhydrate. Berlin Klin Wochschr. 1906;43:978-981.

11. Nubel T, Emre Y, Rabier D, Chadefaux B, Ricquier $\mathrm{D}$, Bouillaud F. Modified glutamine catabolism in macrophages of Ucp 2 knock-out mice. Biochim Biophys Acta. 2008;1777(1):48-54

12. Taegtmeyer H. Cardiac metabolism as a target for the treatment of heart failure. Circulation. 2004;110(8):894-896.

13. Bonnet $\mathrm{S}$, et al. A mitochondria- $\mathrm{K}+$ channel axis is suppressed in cancer and its normalization promotes apoptosis and inhibits cancer growth. Cancer Cell. 2007;11(1):37-51.

14. Paumen MB, Ishida Y, Muramatsu M, Yamamoto $\mathrm{M}$, Honjo T. Inhibition of carnitine palmitoyltransferase I augments sphingolipid synthesis and palmitate-induced apoptosis. J Biol Chem.
1997:272(6):3324-3329.

15. Hernlund E, et al. Potentiation of chemotherapeutic drugs by energy metabolism inhibitors 2-deoxyglucose and etomoxir. Int J Cancer. 2008;123(2):476-483.

16. Giordano A, et al. tBid induces alterations of mitochondrial fatty acid oxidation flux by malonyl-CoAindependent inhibition of carnitine palmitoyltransferase-1. Cell Death Differ. 2005;12(6):603-613.

17. Paumen MB, et al. Direct interaction of the mitochondrial membrane protein carnitine palmitoyltransferase I with Bcl-2. Biochem Biophys Res Commun. 1997;231(3):523-525.

18. Konopleva M, et al. Mechanisms of apoptosis sensitivity and resistance to the $\mathrm{BH} 3$ mimetic ABT-737 in acute myeloid leukemia. Cancer Cell. 2006;10(5):375-388.

19. MacInnes A, et al. The antianginal agent trimetazidine does not exert its functional benefit via inhibition of mitochondrial long-chain 3-ketoacyl coenzyme A thiolase. Circ Res. 2003;93(3):e26-e32.

20. Lopaschuk GD, McNeil GF, McVeigh JJ. Glucose oxidation is stimulated in reperfused ischemic hearts with the carnitine palmitoyltransferase 1 inhibitor, Etomoxir. Mol Cell Biochem. 1989;88(1-2):175-179.

21. Pizer ES, Wood FD, Pasternack GR, Kuhajda FP. Fatty acid synthase (FAS): a target for cytotoxic antimetabolites in HL60 promyelocytic leukemia cells. Cancer Res. 1996;56(4):745-751.

22. Pallasch CP, et al. Targeting lipid metabolism by the lipoprotein lipase inhibitor orlistat results in apoptosis of B-cell chronic lymphocytic leukemia cells. Leukemia. 2008;22(3):585-592.

23. Cabrero A, et al. Increased reactive oxygen species production down-regulates peroxisome proliferator-activated alpha pathway in C2C12 skeletal muscle cells. J Biol Chem. 2002;277(12):10100-10107.

24. Spurway TD, Pogson CI, Sherratt HS, Agius L. Etomoxir, sodium 2-[6-(4-chlorophenoxy)hexyl] oxirane-2-carboxylate, inhibits triacylglycerol depletion in hepatocytes and lipolysis in adipocytes. FEBS Lett. 1997;404(1):111-114.

25. Tabe $Y$, et al. Activation of integrin-linked kinase is a critical prosurvival pathway induced in leukemic cells by bone marrow-derived stromal cells. Cancer Res. 2007;67(2):684-694.

26. Zhang CY, et al. Genipin inhibits UCP2-mediated proton leak and acutely reverses obesity- and high glucose-induced beta cell dysfunction in isolated pancreatic islets. Cell Metab. 2006;3(6):417-427.

27. Kuan CY, et al. A critical role of neural-specific JNK3 for ischemic apoptosis. Proc Natl Acad Sci U S A. 2003;100(25):15184-15189.

28. Leinninger GM, et al. Mitochondria in DRG neurons undergo hyperglycemic mediated injury through Bim, Bax and the fission protein Drp1. Neurobiol Dis. 2006;23(1):11-22.

29. Certo M, et al. Mitochondria primed by death signals determine cellular addiction to antiapoptotic BCL-2 family members. Cancer Cell. 2006;9(5):351-365.

30. Kojima K, et al. MDM2 antagonists induce p53dependent apoptosis in AML: implications for leukemia therapy. Blood. 2005;106(9):3150-3159.

31. Chipuk JE, et al. Direct activation of Bax by p 53 mediates mitochondrial membrane permeabilization and apoptosis. Science. 2004;303(5660):1010-1014.

32. Kojima K, et al. The dual PI3 kinase/mTOR inhibitor PI- 103 prevents $\mathrm{p} 53$ induction by $\mathrm{Mdm} 2$ inhibition but enhances p53-mediated mitochondrial apoptosis in p53 wild-type AML. Lenkemia. 2008;22(9):1728-1736.

33. Jin J, et al. Ceramide generated by sphingomyelin hydrolysis and the salvage pathway is involved in hypoxia/reoxygenation-induced Bax redistribution to mitochondria in NT-2 cells. J Biol Chem. 2008;283(39):26509-26517.

34. Ruffolo SC, Shore GC. BCL-2 selectively interacts with the BID-induced open conformer of BAK, inhibiting BAK auto-oligomerization. J Biol Chem. 2003;278(27):25039-25045.

35. Guan Y, Gerhard B, Hogge DE. Detection, isolation, and stimulation of quiescent primitive leukemic progenitor cells from patients with acute myeloid leukemia (AML). Blood. 2003;101(8):3142-3149.

36. Konopleva $\mathrm{M}$, et al. The anti-apoptotic genes Bcl$\mathrm{X}(\mathrm{L})$ and $\mathrm{Bcl}-2$ are over-expressed and contribute to chemoresistance of non-proliferating leukaemic CD34+ cells. Br J Haematol. 2002;118(2):521-534.

37. Moreno-Sanchez R, Rodriguez-Enriquez S, MarinHernandez A, Saavedra E. Energy metabolism in tumor cells. FEBS J. 2007;274(6):1393-1418.

38. Pecqueur C, et al. Uncoupling protein-2 controls proliferation by promoting fatty acid oxidation and limiting glycolysis-derived pyruvate utilization. FASEB J. 2008;22(1):9-18. 
39. Hirabara SM, et al. Role of fatty acids in the transition from anaerobic to aerobic metabolism in skeletal muscle during exercise. Cell Biochem Funct. 2006;24(6):475-481.

40. Hirabara SM, et al. Acute effect of fatty acids on metabolism and mitochondrial coupling in skeletal muscle. Biochim Biophys Acta. 2006;1757(1):57-66.

41. Young ME, et al. Uncoupling protein 3 transcription is regulated by peroxisome proliferator-activated receptor (alpha) in the adult rodent heart. FASEB J. 2001;15(3):833-845.

42. Chen Z, Lu W, Garcia-Prieto C, Huang P. The Warburg effect and its cancer therapeutic implications. J Bioenerg Biomembr. 2007;39(3):267-274.

43. Chipuk JE, Fisher JC, Dillon CP, Kriwacki RW,
Kuwana T, Green DR. Mechanism of apoptosis induction by inhibition of the anti-apoptotic BCL-2 proteins. Proc Natl Acad Sci U S A. 2008;105(51):20327-20332.

44. de Pablo MA, et al. Palmitate induces apoptosis via a direct effect on mitochondria. Apoptosis. 1999;4(2):81-87.

45. Vickers AE. Characterization of hepatic mitochondrial injury induced by fatty acid oxidation inhibitors. Toxicol Pathol. 2009;37(1):78-88.

46. Vander Heiden MG, Cantley LC, Thompson CB. Understanding the Warburg effect: the metabolic requirements of cell proliferation. Science. 2009;324(5930):1029-1033.

47. Oltersdorf T, et al. An inhibitor of Bcl-2 family pro- teins induces regression of solid tumours. Nature. 2005;435(7042):677-681.

48. Samudio I, et al. A novel mechanism of action of methyl-2-cyano-3,12 dioxoolean-1,9 diene-28oate (CDDO-Me): direct permeabilization of the inner mitochondrial membrane to inhibit electron transport and induce apoptosis. Mol Pharmacol. 2006;69(4):1182-1193.

49. Wilson-Fritch L, et al. Mitochondrial remodeling in adipose tissue associated with obesity and treatment with rosiglitazone. J Clin Invest. 2004;114(9):1281-1289.

50. Ross J, et al. Fatty acid synthase inhibition results in a magnetic resonance-detectable drop in phosphocholine. Mol Cancer Ther. 2008;7(8):2556-2565. 